\title{
Prefetch-Aware Memory Controllers
}

\author{
Chang Joo Lee, Student Member, IEEE, Onur Mutlu, Member, IEEE, \\ Veynu Narasiman, Student Member, IEEE, and Yale N. Patt, Fellow, IEEE
}

\begin{abstract}
Existing DRAM controllers employ rigid, nonadaptive scheduling and buffer management policies when servicing prefetch requests. Some controllers treat prefetches the same as demand requests, and others always prioritize demands over prefetches. However, none of these rigid policies result in the best performance because they do not take into account the usefulness of prefetches. If prefetches are useless, treating prefetches and demands equally can lead to significant performance loss and extra bandwidth consumption. In contrast, if prefetches are useful, prioritizing demands over prefetches can hurt performance by reducing DRAM throughput and delaying the service of useful requests. This paper proposes a new low hardware cost memory controller, called as Prefetch-Aware DRAM Controller (PADC), that aims to maximize the benefit of useful prefetches and minimize the harm caused by useless prefetches. The key idea is to 1) adaptively prioritize between demands and prefetches, and 2) drop useless prefetches to free up memory system resources, based on prefetch accuracy. Our evaluation shows that PADC significantly outperforms previous memory controllers with rigid prefetch handling policies. Across a wide range of multiprogrammed SPEC CPU 2000/2006 workloads, it improves system performance by 8.2 and 9.9 percent on four and eight-core systems while reducing DRAM bandwidth consumption by 10.7 and 9.4 percent, respectively.
\end{abstract}

Index Terms-Memory systems, prefetching, memory controllers, DRAM, multi-core systems.

\section{INTRODUCTION}

$\mathrm{H}$ IGH-PERFORMANCE memory controllers seek to maximize throughput by exploiting row buffer locality. A modern SDRAM bank contains a row buffer that buffers the data of the last accessed memory row. Therefore, an access to the same row (called row-hit) can be serviced significantly faster than an access to a different row (called row-conflict) [18]. Due to this nonuniform access latency, state-of-the-art memory access scheduling policies such as [47], [31], [17] prefer row-hits to row-conflicts to improve DRAM throughput, thereby improving system performance. However, the problem of DRAM access scheduling becomes more challenging when we take prefetch requests into consideration.

Today's microprocessors employ hardware prefetchers to hide long DRAM access latencies. If prefetch requests are accurate and fetch data early enough, prefetching can improve performance. However, even if prefetch accuracy is high, the full benefit of prefetching may not be achieved based on how the DRAM controller schedules the requests. For example, a demand request can delay a prefetch request that could have, otherwise, been serviced very fast in DRAM, e.g., if the prefetch request is a row-hit, while the demand request is a row-conflict. If the prefetch is useful, delaying it by servicing the row-conflict demand request first may not result in the best performance.

- C.J. Lee, V. Narasiman, and Y.N. Patt are with the Department of Electrical and Computer Engineering, The University of Texas at Austin, 1 University Station C0803, Austin, TX 78712-0240.

E-mail: \{cjlee, narasima, patt\}@ece.utexas.edu.

- O. Mutlu is with the Department of Electrical and Computer Engineering, Carnegie Mellon University, 5000 Forbes Avenue, Pittsburgh, PA 152133890.E-mail: onur@cmu.edu.

Manuscript received 26 May 2009; revised 4 Dec. 2009; accepted 12 May 2010; published online 20 Oct. 2010.

Recommended for acceptance by R. Gupta.

For information on obtaining reprints of this article, please send e-mail to: tc@computer.org, and reference IEEECS Log Number TC-2009-05-0227.

Digital Object Identifier no. 10.1109/TC.2010.214.
In addition, prefetching does not always improve and can sometimes degrade performance due to two reasons. First, useless prefetch requests unnecessarily consume valuable off-chip bandwidth and fetch useless data that might displace useful data blocks in processor caches. Second, prefetch requests contend for the same resources (e.g., memory request buffer entries and memory bus bandwidth) as demand (load/store) requests issued by a processing core. As a result, a prefetch request can delay a demand request, which could lead to performance degradation especially if the prefetch is useless. If the interference between prefetch requests and demand requests is not controlled, system performance can degrade because either demand requests or useful prefetch requests can be significantly delayed.

Existing DRAM scheduling policies take two different approaches as to how to treat prefetch requests with respect to demand requests. Some policies [42] regard a prefetch request to have the same priority as a demand request. As noted above, this policy can significantly delay demand requests and cause performance degradation especially if prefetch requests are not accurate. Other policies [9], [14], [7], [35], [36] always prioritize demand requests over prefetch requests so that data known-to-be-needed by the program instructions can be serviced earlier. One might think that always prioritizing demand requests over prefetch requests in the memory controller provides the best performance by eliminating the interference of prefetch requests with demand requests. However, such a rigid demand-over-prefetch prioritization policy does not consider the nonuniform access latency of the DRAM system (row-hits versus row-conflicts). A row-hit prefetch request can be serviced much more quickly than a row-conflict demand request. Therefore, servicing the row-hit prefetch request first provides better DRAM throughput and can improve system performance compared to servicing the row-conflict demand request first. 


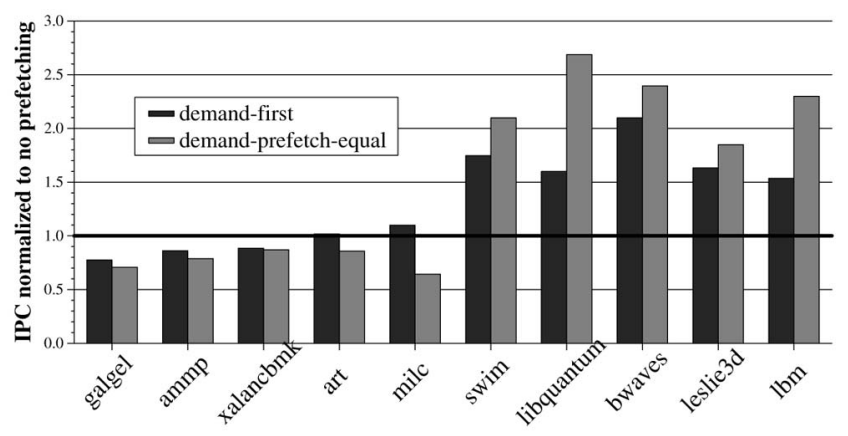

Fig. 1. Normalized performance of a stream prefetcher with two different DRAM scheduling policies.

Fig. 1 provides supporting data to demonstrate this. This figure shows performance impact of an aggressive stream prefetcher [36], [39] when used with two different memory scheduling policies for 10 SPEC 2000/2006 benchmarks on a single processor described in Section 5 . The vertical axis is retired instructions per cycle (IPC) normalized to the IPC on a processor with no prefetching. One policy, demand-prefetchequal, does not differentiate between demand and prefetch requests. This policy is the same as the First Ready-First Come First Serve (FR-FCFS) policy that prioritizes requests as follows: 1) row-hit requests over all others and 2) older requests over younger requests [31]. As a result, DRAM throughput is maximized. The other policy, demand-first, prioritizes demand requests over prefetch requests. Prefetch requests to a bank are not scheduled until all the demand requests to the same bank are serviced. Within a set of outstanding demand or prefetch requests, the policy uses the same prioritization rules as the FR-FCFS policy. As a result, this policy does not maximize overall DRAM throughput because it prefers row-conflict demand requests over row-hit prefetch requests. ${ }^{1}$

The results show that neither of the two policies provides the best performance for all applications. For the leftmost five applications, prioritizing demands over prefetches results in better performance than treating prefetches and demands equally. In these applications, a large fraction (70 percent for demand-prefetch-equal and 59 percent for demand-first) of the generated stream prefetch requests are useless. Therefore, it is important to prioritize demand requests over prefetches. In fact, for art and milc, servicing the demand requests with higher priority is critical to make prefetching effective. Prefetching improves performance of these two applications by 2 and 10 percent, respectively, with the demand-first scheduling policy, whereas it reduces their performance by 14 and 36 percent with the demand-prefetch-equal policy.

On the other hand, for the rightmost five applications, we observe the exact opposite behavior. Equally treating demand and prefetch requests provides significantly higher performance than prioritizing demands over prefetches. In particular for libquantum, the demand-prefetch-equal policy allows the prefetcher to provide 169 percent performance improvement in contrast to the 60 percent performance

1. For completeness, we also implemented another policy, prefetch-first, that always prioritizes prefetch requests over demand requests. This policy provides the worst performance (5.8 percent IPC degradation compared to demand-first policy) on all the benchmarks. improvement it provides with the demand-first scheduling policy. This is because prefetch requests in libquantum are very accurate (almost 100 percent of them are useful). Maximizing DRAM throughput by preferring row buffer hits in the DRAM system regardless of whether a memory request is a demand or a prefetch request allows for more efficient bandwidth utilization and improves the timeliness (and the coverage) of prefetches thereby improving system performance. ${ }^{2}$ These results show that DRAM scheduling policies with rigid prioritization rules among prefetch and demand requests cannot provide the best performance and may even cause prefetching to degrade performance.

Note that even though the DRAM scheduling policy has a significant impact on performance provided by prefetching, prefetching sometimes degrades performance regardless of the DRAM scheduling policy. For example, galgel, ammp, and xalancbmk suffer significant performance loss with prefetching because a large fraction $(69,94$, and 91 percent) of the prefetches are not needed by the program. The negative performance impact of these useless prefetch requests cannot be mitigated solely by a demand-first scheduling policy because useless prefetches 1 ) occupy memory request buffer entries in the memory controller until they are serviced, 2) occupy DRAM bandwidth while they are being serviced, and 3) cause cache pollution by evicting possibly useful data from the processor caches after they are serviced. As a result, useless prefetches could delay the servicing of demand requests and could result in additional demand requests. In essence, useless prefetch requests can deny service to demand requests because the DRAM controller is not aware of the usefulness of prefetch requests in its memory request buffer. To prevent this, the memory controller should intelligently manage the memory request buffer between prefetch and demand requests.

Our goal in this paper is to design an adaptive DRAM controller that is aware of prefetching. We propose a memory controller that adaptively controls the interference between prefetch and demand requests to improve system performance. Our controller aims to maximize the benefits of useful prefetches and minimize the harm of useless prefetches. To do so, it employs two techniques to manage both memory bandwidth and memory request buffers: based on the runtime behavior (accuracy) of the prefetcher, it 1 ) adaptively decides whether or not to prioritize demand requests over prefetch requests, and 2) decides whether or not to drop likely-useless prefetches from the memory request buffer.

We evaluate our Prefetch-Aware DRAM Controller on a wide variety of benchmarks and systems, and find that it consistently outperforms previous DRAM controllers that rigidly handle prefetches on both single-core and multicore (two, four, and eight-core) systems. Our controller improves single-core performance by up to 68 percent (on average, 4.3 percent) for the 55 SPEC $2000 / 2006$ benchmarks when compared to a controller that employs the demand-first policy. Our mechanism also improves system performance (i.e., weighted speedup) for 54 SPEC workloads by 8.4 percent on a two-core system, for 32 workloads by 8.2 percent on a

2. Improving DRAM throughput improves prefetch coverage by reducing the probability that a useful prefetch is not issued into the memory system because the memory request buffer is full. We explain this in more detail in Section 6.1. To show how our mechanism improves DRAM throughput for useful requests compared to rigid policies, we also analyze row-hit rates for demand and useful prefetch requests with the rigid policies and our mechanism in detail in Section 6.1.2. 
four-core system, and for 21 SPEC workloads by 9.9 percent on an eight-core system while also reducing memory bandwidth consumption by 10.0, 10.7, and 9.4 percent for two, four, and eight-core systems, respectively. We show that our controller has low implementation complexity and storage cost, requiring only $4.25 \mathrm{~KB}$ of storage in a four-core system.

Contributions. To our knowledge, this is the first paper that comprehensively and adaptively incorporates prefetch awareness into the memory controller's scheduling and request buffer management policies. We make the following contributions:

1. We show that performance of a prefetcher significantly depends on how prefetch requests are handled by the memory controller with respect to demand requests. Rigid memory scheduling policies that treat prefetches and demands equally or that always prioritize demands can either cause significant losses in system performance or not achieve the best performance for all applications.

2. We propose a low hardware cost memory controller design that dynamically adapts its prioritization policy between demand and prefetch requests based on how accurate the prefetcher is in a given program phase. This mechanism achieves high performance by improving both DRAM throughput and prefetch timeliness/coverage.

3. We propose a simple mechanism that reduces the interference of useless prefetches with demand requests by proactively removing the likely-useless prefetches from the memory request buffer. This mechanism efficiently reduces the buffer, bandwidth, and cache space resources consumed by useless prefetches thereby improving both performance and bandwidth efficiency.

4. We show that the proposed adaptive scheduling and buffer management mechanisms interact positively. Our Prefetch-Aware DRAM Controller (PADC) that comprises both mechanisms significantly improves performance and bandwidth efficiency on both single-core and multicore systems. In addition, our proposal is very effective for a variety of prefetching algorithms including stream, PC-based stride, CZone/Delta Correlation (C/DC), and Markov prefetching.

5. We comprehensively evaluate performance and DRAM bus traffic of PADC on various DRAM and last-level cache configurations. We also compare and incorporate PADC with other mechanisms such as hardware prefetch filtering, feedback directed prefetching, permutation-based page interleaving, and runahead execution. Our results show that PADC not only outperforms but also complements all of these previous performance enhancement mechanisms.

\section{BACKGROUND}

\subsection{DRAM Systems and Scheduling}

We provide background into DRAM organization and characteristics to motivate the problem and the solution proposed in this section. Readers can find more information on detailed DRAM structures and DRAM system organization in other works [18], [3], [31].

An SDRAM system consists of multiple banks that can be accessed independently. Each DRAM bank is a twodimensional array comprising rows and columns of DRAM cells. A row contains a fixed-size block of data (usually several Kilobytes). Each bank has a row buffer (or sense amplifier). The row buffer can store only a single row. To access a location in a row, the entire row needs to be brought into the row buffer (i.e., sense-amplified). In essence, the row buffer acts as storage that buffers the most recently accessed row in the DRAM bank. A DRAM access can be done only by reading (writing) data from (to) the row buffer using a column address.

There are three possible sequential commands that need to be issued to a DRAM bank in order to access data. A memory controller may issue 1) a precharge command to precharge the bank's bitlines, 2) an activate command to open a row into the row buffer with the row address, and then, 3) a read/write command to access the row buffer with the column address. After the completion of an access, the DRAM controller can either keep the row open in the row buffer (open-row policy) or close the row buffer with a precharge command (closed-row policy). The latency of a memory access to a bank varies depending on the state of the row buffer and the address of the request as follows:

1. Row-hit: The row address of the memory access is the same as the address of the opened row. Data can be read from/written to the row buffer by a read/write command; therefore, the total latency is only the $\mathrm{read} / \mathrm{write}$ command latency.

2. Row-conflict: The row address of the memory access is different from the address of the opened row. The memory access needs a precharge, an activate, and a $\mathrm{read} /$ write command sequentially. The total latency is the sum of all three command latencies.

3. Row-closed: There is no valid data in the row buffer (i.e., closed). The access needs an activate command, and then, a read/write command. The total latency is the sum of these two command latencies.

DRAM access time is the shortest in the case of a row-hit $[18]^{3}{ }^{3}$ Therefore, a memory controller can try to maximize DRAM data throughput by maximizing the hit rate in the row buffer, i.e., by first scheduling row-hit requests among all the requests in the memory request buffer. Previous work [31] introduced the commonly employed FR-FCFS policy which prioritizes requests such that it services 1) row-hit requests first and 2) all else being equal, older requests first. This policy was shown to provide the best average performance in systems that do not employ hardware prefetching [31], [17]. Unfortunately, this policy is not aware of the interaction and interference between demand and prefetch requests in the DRAM system, and therefore, treats demand and prefetch requests equally.

3. The row-hit latency is about one-third of the row-conflict latency in contemporary SDRAM systems. For example, the row-hit and row-conflict latencies are 12.5 and $37.5 \mathrm{~ns}$, respectively, for a 2 Gbit DDR3 SDRAM chip [18]. The row-closed latency is $25 \mathrm{~ns}$. We use the open-row policy throughout the paper since it increases the possibility to improve DRAM throughput. We also evaluate the closed-row policy in Section 6.8 for completeness. 


\subsection{Hardware Prefetchers}

In most of our experiments, we use a stream prefetcher similar to the one used in IBM's POWER 4/5 [39]. Stream prefetchers are commonly used in many processors [39], [6], [12] since they do not require significant hardware cost and work well for a large number of applications. They try to identify sequential streams of data that the application needs by closely monitoring and recording previous sequential accesses. Once a stream is identified, prefetch requests are sent out for data further down the stream so that when the processor actually demands the data, it will already be in the cache. As such, stream prefetchers are likely to generate many useful row-hit prefetch requests which PADC can take advantage of. Therefore, we achieve significant performance improvements with PADC combined with a stream prefetcher. Our implementation of a stream prefetcher is explained in the next section.

We also evaluated our mechanism with other prefetchers. The stride prefetcher [1] is similar to the stream prefetcher, but instead of identifying only sequential streams, it detects sequences of addresses that differ by a constant value (stride) and generates prefetch requests that continue in the stride pattern. The Markov Prefetcher [9], a correlationbased prefetcher, records in a large table the cache miss addresses that follow a cache miss. If the same miss occurs again, the table is accessed, and prefetch requests to the recorded next address(es) are generated. We also evaluated PADC with the previously proposed C/DC prefetcher [27]. This prefetcher divides the address space statically into fixed-size regions (CZones) and finds patterns among miss addresses within a region. It uses delta correlations to detect patterns more complex than simple strides and generates prefetch requests that follow the detected pattern. Note that all of these prefetchers can also generate a significant number of row-hit prefetch requests especially for streaming/striding address/correlation patterns. In the results section, we show that PADC can improve performance when combined with any of these prefetchers and is not restricted to working with only a stream prefetcher.

\subsection{Stream Prefetcher}

We discuss an implementation of an aggressive stream prefetcher (similar to the one used in the IBM POWER4/5 [39]) used to collect most of our experimental results. The stream prefetcher we use for the evaluations is the best performing among the large set of prefetchers we examined. It improves IPC by 20 percent, on average, compared to no prefetching for the 55 SPEC 2000/2006 benchmarks when employed with a demand-first scheduling policy. The stream prefetcher prefetches cache lines into the L2 cache.

Each stream entry in the stream prefetcher monitors a range of consecutive cache line addresses beginning at a starting pointer $(\mathrm{S})$ and ending at $S$ plus a prefetch distance (D). We call this range of consecutive cache line addresses a monitoring region. We also associate with the stream prefetcher a prefetch degree $(\mathrm{N})$. When a new (not part of an existing stream) cache miss occurs, a stream entry is allocated and the cache line address is recorded as $S$. When subsequent cache accesses (both cache hits and misses) within a small distance from $S$ occur, the direction of prefetching is determined and the monitoring region is set up starting with $\mathrm{S}$ and ending with $\mathrm{S}+\mathrm{D}$. If an L2 cache access happens within the monitoring region, $\mathrm{N}$ consecutive prefetch requests from cache line address $S+D+1$ to $S+D+N$ are sent to the memory system. After prefetches are sent, the monitoring region is shifted in the direction of prefetching by the number of the requests sent.

The timeliness and aggressiveness of the stream prefetcher are functions of both $\mathrm{D}$ and $\mathrm{N}$. Larger values for $\mathrm{D}$ and $\mathrm{N}$ result in more prefetch requests that are far ahead of the current access stream so that more latency can be hidden if the prefetches are accurate. On the other hand, overly aggressive values for $\mathrm{D}$ and $\mathrm{N}$ can prevent demand requests from being serviced since prefetches can fill up a larger fraction of the memory system buffer resources. We found that a prefetch degree of 4 and a prefetch distance of 64 result in the best performance on our single-core system described in Section 5.

Since the stream prefetcher tries to fetch consecutive cache lines from a region, it is likely that it will generate many row-hit prefetch requests. If the row buffer locality of these prefetch requests is not exploited intelligently by the DRAM scheduling policy, DRAM throughput and system performance can degrade.

\section{Motivation: Rigid Prefetch Scheduling in DRAM SYSTEMS}

None of the existing DRAM scheduling policies [9], [31], [42], [14], [35], [36] take into account both the nonuniform nature of DRAM access latencies and the behavior of prefetch requests, i.e., whether they are useful or useless. We illustrate why a rigid, nonadaptive prefetch scheduling policy degrades performance in Fig. 2. Consider the example in Fig. 2a, which shows three outstanding memory requests (to the same bank) in the memory request buffer. Row $\mathrm{A}$ is currently open in the row buffer of the bank. Two requests are prefetches (to addresses $X$ and $Z$ ) that access row $\mathrm{A}$, while one request is a demand request (to address $\mathrm{Y}$ ) that accesses row B. This example abstracts away many details of the DRAM system (such as DRAM bus/bank timing constraints and queuing delays) for ease of understanding. We omit them from the figure to clearly illustrate the concept of rigid prefetch scheduling in the DRAM controller. However, our simulations faithfully model all details of the DRAM system.

For Fig. 2b, assume that the processor needs to load addresses $Y, X$, and $Z$ in a serial fashion (i.e., the prefetches are useful) and the computation between each load instruction takes a fixed and small number of cycles (25 in the figure) that is significantly smaller than the DRAM access latency. We assume that processor execution takes a small number of cycles because previous studies [24], [11] have shown that most of the execution time is dominated by DRAM access latency. Fig. $2 b$ shows the service timeline of the requests in DRAM and the resulting execution timeline of the processor for two different memory scheduling policies: demand-first and demand-prefetch-equal. With the demand-first policy (top), the row-conflict demand request is satisfied first which causes the prefetch of address $X$ to incur a row-conflict as well. The subsequent prefetch request to $Z$ is a row-hit 


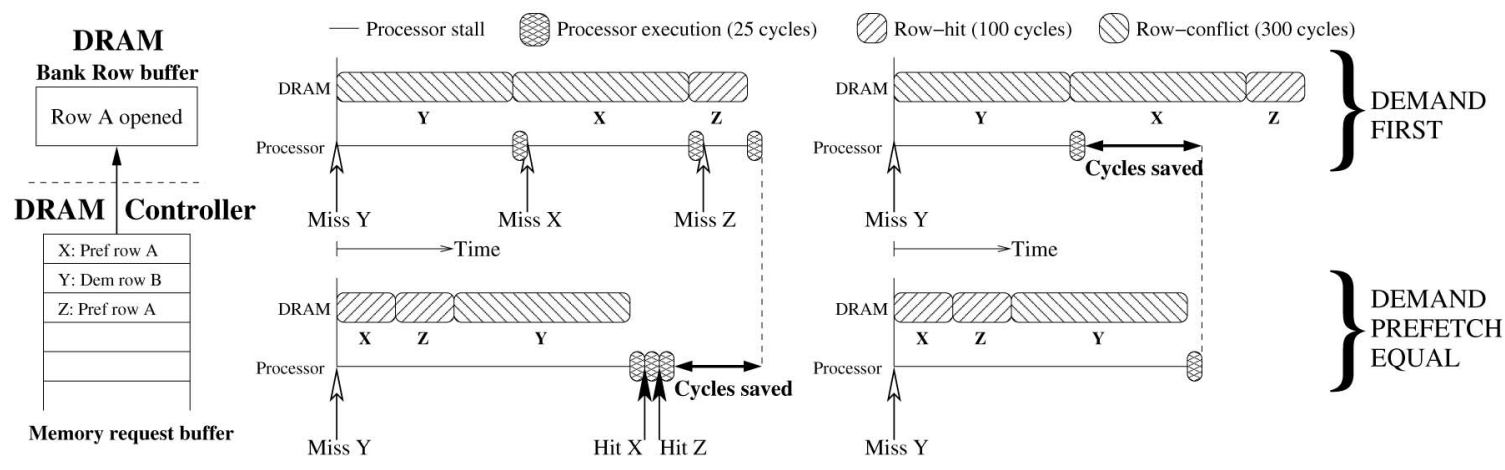

(a)

(b)

(c)

Fig. 2. Performance impact of demand-first versus demand-prefetch-equal policy. (a) DRAM and controller state. (b) Timeline when prefetches are useful. (c) Timeline when prefetches are useless.

because the prefetch of $X$ opens row $A$. As a result, the processor first stalls for approximately two row-conflict latencies (except for a small period of execution) to access address $Y$, and then, to access address $X$. The processor then stalls for an additional row-hit latency (again with the exception of another small period of execution) since it requires the data prefetched from address $\mathrm{Z}$. The total execution time is the sum of two row-conflict latencies and one row-hit latency plus a small period of processor execution (the other computation periods are hidden) which total to 725 cycles in this example.

With the demand-prefetch-equal policy (bottom), the row-hit prefetch requests to $X$ and $Z$ are satisfied first. Then, the row-conflict demand request to $Y$ is serviced. The processor stalls until the demand request to $\mathrm{Y}$ is serviced. However, once the demand request is serviced, the processor does not stall any more because the memory requests to the other addresses it needs ( $X$ and $Z$ ) have already been serviced and placed into the cache. The processor only needs to perform the computations between the load instructions and finds that loads to $X$ and $Z$ hit in the cache. The resulting total execution time is the sum of two row-hit latencies, one row-conflict latency, and the latency to execute the computation between each load instruction for a total of only 575 cycles in this example. Hence, treating prefetches and demands equally can significantly improve performance when prefetch requests are useful. We observe that the stream prefetcher generates very accurate prefetch requests for many memoryintensive applications such as libquantum, swim, and leslie3d. For these applications, the demand-prefetch-equal memory scheduling policy increases prefetch timeliness by increasing DRAM throughput, and therefore, improves performance significantly, as shown in Fig. 1.

However, prefetch requests might not always be useful. In the example of Fig. 2a, assume that the processor needs to load only address $Y$ but still generates useless prefetches to addresses $X$ and $Z$. Fig. 2c shows the service timeline of the requests and the resulting execution timeline of the processor for the two different memory scheduling policies. With the demand-first policy (top), the processor needs to stall only for a single row-conflict latency that is required to service the demand request to $Y$, and therefore, the total execution time is 325 cycles. On the other hand, with the demand-prefetch-equal policy, the processor needs to stall additional cycles since $X$ and $Z$ are serviced (even though they are not needed) before $Y$. It takes two row-hit requests to service the useless prefetches to $X$ and $Z$ and one rowconflict request to service the demand request to $Y$. The resulting execution time is 525 cycles. Hence, treating prefetches and demands equally can significantly degrade performance when prefetch requests are useless. In fact, our experimental data in Fig. 1 showed that treating demands and prefetches equally in applications where most of the prefetches are useless causes prefetching to degrade performance by up to 36 percent (for milc).

These observations illustrate that 1) DRAM scheduling policies that rigidly prioritize between demand and prefetch requests without taking into account the usefulness of prefetch requests can either degrade performance or fail to provide the best possible performance, and 2) the effectiveness of a particular prefetch prioritization mechanism significantly depends on the usefulness of prefetch requests. Based on these observations, to improve the effectiveness of prefetching, we aim to develop an adaptive DRAM scheduling policy that dynamically changes the prioritization order of demands and prefetches by taking into account the usefulness of prefetch requests.

\section{Mechanism: Prefetch-Aware DRAM CONTROLLER}

Our PADC consists of two components as shown in Fig. 3: An Adaptive Prefetch Scheduling (APS) unit and an Adaptive Prefetch Dropping (APD) unit. APS tries to 1) maximize the benefits of useful prefetches by increasing DRAM throughput and 2) minimize the harm of useless prefetches by delaying their DRAM service, and hence, reducing their interference with demand and useful prefetch requests. APD cancels useless prefetch requests effectively while preserving the benefits of useful prefetches. Both APS and APD are driven by the measurement of each processing core's prefetch accuracy in a multicore system. Before explaining how each component works, we explain how prefetch accuracy is measured for each core. 


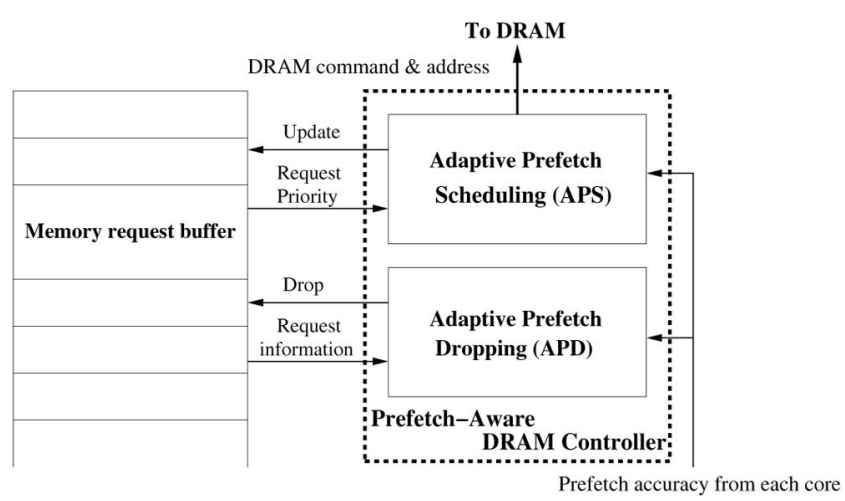

Fig. 3. Prefetch-aware DRAM controller.

\subsection{Prefetch Accuracy Measurement}

We measure the prefetch accuracy for an application running on a particular core over a certain time interval. The accuracy is reset once the interval has elapsed so that the mechanism can adapt to the phase behavior of prefetching. To measure the prefetch accuracy of each core, the following hardware support is required:

1. Prefetch $(\mathrm{P})$ bit per L2 cache line and memory request buffer entry: For memory request buffer entries, this bit indicates whether or not the request was generated by the prefetcher. It is set when a new memory request is generated by the prefetcher, and reset when the processor issues a demand request to the same cache line, while the prefetch request is still in the memory request buffer. For cache lines, this bit indicates whether or not a cache line was brought into the cache by a prefetch request. It is set when the line is filled (only if the prefetch bit of the request is set) and is reset when a cache hit to the same line occurs.

2. Prefetch Sent Counter (PSC) per core: This counter keeps track of the total number of prefetch requests sent by a core. It is incremented whenever a prefetch request is sent to the memory request buffer by the core.

3. Prefetch Used Counter (PUC) per core: This counter keeps track of the number of prefetches that are useful. It is incremented when a prefetched cache line is used (cache hit) by a demand request and also when a demand request matches a prefetch request already in the memory request buffer.

4. Prefetch Accuracy Register (PAR) per core: This register stores the prefetch accuracy measured every time interval. PAR is computed by dividing PUC by PSC.

At the end of every time interval, PAR is updated with the prefetch accuracy calculated during that interval and PSC and PUC are reset to 0 to calculate the accuracy for the next interval. The PAR values for each core are fed into the Prefetch-Aware DRAM Controller which then uses the values to guide its scheduling and memory request buffer management policies.

\subsection{Adaptive Prefetch Scheduling}

APS changes the priority of demand/prefetch requests from a processing core based on the prefetch accuracy estimated for that core. The basic idea is to 1) treat useful prefetch requests the same as demand requests so that useful prefetches can be serviced faster by maximizing DRAM throughput, and 2) give demand and useful prefetch requests a higher priority than useless prefetch requests so that useless prefetch requests do not interfere with demand and useful prefetch requests.

If the prefetch accuracy is greater than or equal to a certain threshold, promotion_threshold, all of the prefetch requests from that core increase in priority and are treated the same as demand requests. We call such prefetch requests and all demand requests critical requests. If the estimated prefetch accuracy of a core is less than promotion_threshold, then demand requests of that core are prioritized over prefetch requests. We call such prefetch requests noncritical requests.

The essence of our proposal is to prioritize critical requests over noncritical ones in the memory controller while preserving DRAM throughput. To accomplish this, our mechanism prioritizes memory requests in the order shown in Rule 1. Each prioritization decision in this set of rules is described in further detail below.

Rule 1. Adaptive prefetch scheduling

1. Critical request $(\mathbf{C})$ : Critical requests are prioritized over all other requests.

2. Row-hit request (RH): Row-hit requests are prioritized over row-conflict requests.

3. Urgent request (U): Demand requests generated by cores with low prefetch accuracy are prioritized over other requests.

4. Oldest request (FCFS): Older requests are prioritized over younger requests.

First, critical requests (useful prefetches and demand requests) are prioritized over others. This delays the scheduling of noncritical requests most of which are likely to be useless prefetches. As a result, useless prefetches are prevented from interfering with demands and useful prefetches.

Second, row-hit requests are prioritized over others. This increases the row buffer locality for demand and useful prefetch requests and maximizes DRAM throughput as much as possible.

Third, demand requests from cores whose prefetch accuracy is less than promotion_threshold are prioritized. We call such requests urgent requests. Intuitively, this rule tries to give a boost to the demand requests of a core with low prefetch accuracy over the demand and useful prefetch requests of cores with high prefetch accuracy. We do this for two reasons. First, if a core has high prefetch accuracy, its prefetch requests will be treated the same as the demand requests of another core with low prefetch accuracy (due to the critical request first prioritization rule). Doing so risks starving the demand requests of the core with low prefetch accuracy resulting in performance degradation since many critical requests from the core with high prefetch accuracy (demand + prefetch requests) will contend with the critical requests from the core with low prefetch accuracy (demand requests only). To avoid such starvation and performance degradation, we boost the demand requests of the core with low prefetch accuracy. Second, performance of a core with low prefetch accuracy is already affected negatively by the 


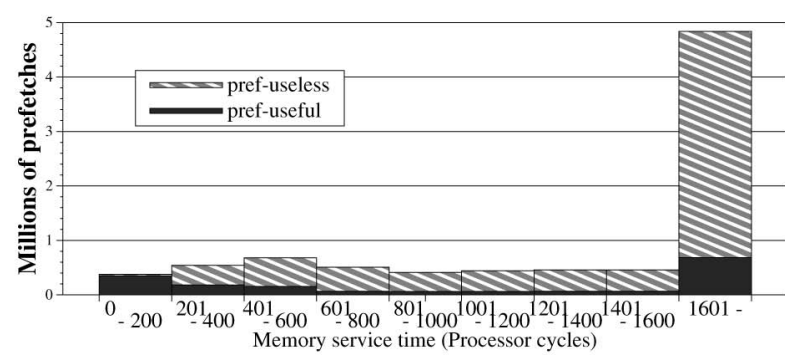

(a)

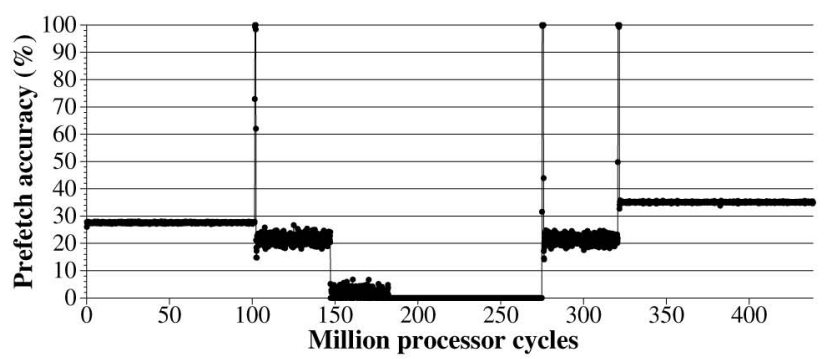

(b)

Fig. 4. Example of behavior of prefetches for milc. (a) Prefetch service time with demand-first policy. (b) Prefetch accuracy every $100 \mathrm{~K}$ cycles.

useless prefetches. By prioritizing the demand requests of such cores over the requests of other cores, we aim to help performance of cores that are already losing performance due to poor prefetcher behavior. We further discuss the effect of prioritizing urgent requests in Section 6.3.4.

Finally, if all else is equal, older requests have priority over younger requests.

\subsection{Adaptive Prefetch Dropping}

APS naturally delays (just like the demand-first policy) the DRAM service of prefetch requests from applications with low prefetch accuracy by making the prefetch requests noncritical as described in Section 4.2. Even though this reduces the interference of useless requests with useful requests, it cannot get rid of all of the negative effects of useless prefetch requests (bandwidth consumption and cache pollution) because such requests will eventually be serviced. As such, APS by itself cannot eliminate all of the negative aspects of useless prefetches. Our second scheme, $\mathrm{APD}$, aims to overcome this limitation by proactively removing old prefetch requests from the request buffer if they have been outstanding for a long period of time. The key insight is that if a prefetch request is old (i.e., has been outstanding for a long time), it is likely to be useless and dropping it from the memory request buffer eliminates the negative effects, the useless request might cause in the future. We first describe why old prefetch requests are likely to be useless based on empirical measurements.

Why are old prefetch requests likely to be useless? Fig. 4a shows the memory service time (from entry into the memory request buffer to entry into the L2 fill buffer) of both useful and useless prefetches for milc using the demand-first scheduling policy. Note that we show detailed data for only milc but found similar behavior in all applications. The graph is a histogram with nine latency intervals measured in processor cycles. Each bar indicates the number of useful/ useless prefetch requests whose memory service time was within that interval. Fifty-six percent of all prefetches have a service time greater than 1,600 processor cycles, and 86 percent of these prefetches are useless. Useful prefetches tend to have a shorter service time than useless prefetches (1,486 cycles compared to 2,238 cycles, on average, for milc). This is because a prefetch request that is waiting in the request buffer becomes a demand request ${ }^{4}$ if the processor sends a demand request for the same address, while the prefetch request is still in the buffer. Such useful prefetches

4. A prefetch request that is hit by a demand request in the memory request buffer becomes a real demand request. However, we count it as a useful prefetch throughout the paper since it was first requested by the prefetcher rather than the processing core. that are hit by demand requests will be serviced earlier by the demand-first prioritization policy. Therefore, useful prefetches, on average, experience a shorter service time than useless prefetches. This is also true when we apply APS since it prioritizes critical requests over noncritical requests.

Mechanism. The observation that old prefetch requests are likely to be useless motivates us to remove a prefetch request from the request buffer if the prefetch is old enough. Our proposal, APD, monitors prefetch requests for each core and invalidates any prefetch request that has been outstanding in the memory request buffer for longer than drop_threshold cycles. We adjust drop_threshold based on the prefetch accuracy for each core measured in the previous time interval. If the prefetch accuracy in the interval is low, our mechanism uses a relatively low value for drop_threshold so that it can quickly remove useless prefetches from the request buffer. If the prefetch accuracy is high in the interval, our mechanism uses a relatively high value for drop_threshold so that it does not prematurely remove useful prefetches from the request buffer. By removing useless prefetches, APD saves resources such as request buffer entries, DRAM bandwidth, and cache space, which can instead be used for critical requests (i.e., demand and useful prefetch requests) rather than being wasted on useless prefetch requests. Note that APD interacts positively with APS since APS naturally delays the service of useless (noncritical) requests so that the APD unit can completely remove them from the memory system thereby freeing up request buffer entries and avoiding unnecessary bandwidth consumption.

Determining drop_threshold. Fig. $4 \mathrm{~b}$ shows the runtime behavior of the stream prefetcher accuracy for milc, an application that suffers from many useless prefetches. Prefetch accuracy was measured as described in Section 4.1, using an interval of $100 \mathrm{~K}$ cycles. The figure clearly shows that prefetch accuracy can have very strong phase behavior. From 150 million to 275 million cycles, the prefetch accuracy is very low (close to 0 percent), implying that many useless prefetch requests were generated during this time. Since almost all prefetches are useless during this period, we would like to be able to quickly drop them. Our mechanism accomplishes this using a low value for drop_threshold. On the other hand, we would want drop_threshold to be much higher during periods of high prefetch accuracy. Our evaluation shows that a simple four-level drop_threshold adjusted dynamically can effectively eliminate useless prefetch requests from the memory system while keeping useful prefetch requests in the memory request buffer. 


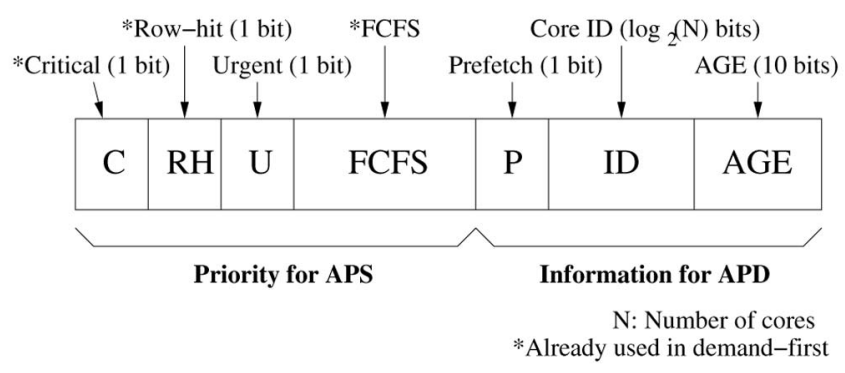

Fig. 5. Memory request field for PADC.

\subsection{Support for Multithreaded Applications on CMP Systems}

Throughout the paper, we assume that each core of a CMP system runs one single-threaded application. However, PADC can also work when multiple multithreaded applications run on a CMP system. This requires a very simple modification in accounting for prefetch requests: instead of associating a prefetch request with the core that generates it, we associate it with the application that generates it. Hence, prefetch requests belonging to different threads in an application (possibly running on different cores) will all contribute to the same prefetch accuracy measurement for the entire multithreaded application. Based on this prefetcher accuracy computed for the entire application, PADC can dynamically prioritize the demand and prefetch requests coming from threads of that application regardless of which core the requests are coming from. Likewise, PADC can also delay and drop likely-useless prefetch requests coming from the threads of an application whose prefetcher accuracy for the entire application is low.

\subsection{Implementation and Hardware Cost of a Prefetch-Aware DRAM Controller}

An implementation of PADC requires storing additional information in each memory request buffer entry to support the priority and aging information needed by APS and APD. The required additional information (in terms of the fields added to each request buffer entry) is shown in Fig. 5 .

The $\mathrm{C}$ (as prefetch bit), $\mathrm{RH}$, and FCFS fields are already used in the baseline demand-first FR-FCFS policy to indicate prefetch status (i.e., demand or prefetch), row-hit status, and arrival time of the request. Therefore, the only additional fields are U, P, ID, and AGE, which indicate the urgency, prefetch status, core ID, and age of the request. In each DRAM cycle, the priority encoder logic chooses the highest priority request using the priority fields $(C, R H, U$, and FCFS) in the order as shown in Fig. 5.

The APD unit removes a prefetch request from the memory request buffer if the request is older than the drop_threshold of the core that generated the request. It does not remove a prefetch request (which is not scheduled for DRAM service) until it ensures that the prefetch cannot be matched by a demand request. This is accomplished by invalidating the MSHR entry of the prefetch request before actually dropping it. The APD unit knows if a request is a prefetch and also which core it belongs to from the $\mathrm{P}$ and ID fields. The AGE field of each request entry keeps track of
TABLE 1

Hardware Cost of Prefetch-Aware DRAM Controller $\left(N_{\text {cache }}\right.$ : Number of Cache Lines Per Core, $N_{\text {core }}$ : Number of Cores, and $N_{\text {req }}$ : Number of Memory Request Buffer Entries)

\begin{tabular}{|c||c|c|c|}
\hline & Bit field & Count & Cost (bits) \\
\hline \hline \multirow{4}{*}{ Prefetch accuracy } & P (1 bit) & $N_{\text {cache }} \times N_{\text {core }}+N_{\text {req }}$ & $N_{\text {cache }} \times N_{\text {core }}+N_{\text {req }}$ \\
\cline { 2 - 4 } & PSC (16 bits) & $N_{\text {core }}$ & $N_{\text {core }} \times 16$ \\
\cline { 2 - 4 } & PUC (16 bits) & $N_{\text {core }}$ & $N_{\text {core }} \times 16$ \\
\cline { 2 - 4 } & PAR (8 bits) & $N_{\text {core }}$ & $N_{\text {core }} \times 8$ \\
\hline APS & U (1 bit) & $N_{\text {req }}$ & $N_{\text {req }}$ \\
\hline \multirow{2}{*}{ APD } & ID (log $N_{\text {core }}$ bits) & $N_{\text {req }}$ & $N_{\text {req }} \times \log _{2} N_{\text {core }}$ \\
\cline { 2 - 4 } & AGE (10 bits) & $N_{\text {req }}$ & $N_{\text {req }} \times 10$ \\
\hline
\end{tabular}

the age of the request. APD compares the AGE of the request to the corresponding core's drop_threshold and removes the request accordingly. Note that the estimation of the age of a request does not need to be highly accurate. For example, the AGE field is incremented every 100 processor cycles for our evaluation.

The hardware storage cost required for our implementation of the PADC is shown in Table 1. Note that the storage cost for PADC linearly increases with the number of cores, request buffer entries, and cache lines. The storage cost for our four-core CMP system described in Section 5 is shown in Table 2. The total storage cost is only 34,720 bits $(\sim 4.25 \mathrm{~KB})$ which is equivalent to only 0.2 percent of the L2 cache data storage in our baseline processor. Note that the Prefetch bit $(\mathrm{P})$ per cache line accounts for over $4 \mathrm{~KB}$ of storage by itself ( $\sim 95$ percent of the total required storage). Many previous proposals [5], [32], [45], [46], [36] already use a prefetch bit for each cache line. If a processor already employs prefetch bits in its cache, the total storage cost of our prefetch-aware DRAM controller is only 1,824 bits ( 228 B). Note that the overhead of prefetch bits can be reduced by using set sampling [29], i.e., associating prefetch bits with only a selected number of sets.

\section{Methodology}

\subsection{Metrics}

We define the metrics used for experimental evaluation in this section. Bus traffic is the number of cache lines transferred over the bus during the execution of a workload. It comprises the cache lines brought in from demand, useful prefetch, and useless prefetch requests. We define Prefetch accuracy (ACC) and coverage (COV) as follows:

TABLE 2

Hardware Cost of PADC on Four-Core System

\begin{tabular}{|c||c|r|}
\hline & Bit field & Cost (bits) \\
\hline \hline \multirow{3}{*}{ Prefetch accuracy } & P & 32,896 \\
\cline { 2 - 3 } & PSC & 64 \\
\cline { 2 - 3 } & PUC & 64 \\
\cline { 2 - 3 } & PAR & 32 \\
\hline \multirow{2}{*}{ APS } & U & 128 \\
\hline \multirow{2}{*}{ APD } & ID & 256 \\
\cline { 2 - 3 } & AGE & 1,280 \\
\hline \hline \multicolumn{2}{|c|}{ Total storage cost for the 4-core system in Section 5 } & 34,720 \\
\hline \multicolumn{2}{|c|}{ Total storage cost as a fraction of the L2 cache capacity } & $0.2 \%$ \\
\hline
\end{tabular}


TABLE 3

Baseline Configuration Per Core

\begin{tabular}{|c|l|}
\hline Execution Core & $\begin{array}{l}\text { 6GHz, Out of order, 15 (fetch, decode, rename stages) stages, decode/retire up to 4 instructions, issue/execute up to 8 microinstructions; } \\
\text { 256-entry reorder buffer; 32-entry load-store queue; 256 physical registers }\end{array}$ \\
\hline \multirow{2}{*}{ Front End } & $\begin{array}{l}\text { Fetch up to 2 branches; 4K-entry BTB; 64-entry return address stack; } \\
\text { Hybrid branch predictor: 64K-entry gshare [16] and 64K-entry PAs predictor [41] with 64K-entry selector }\end{array}$ \\
\hline \multirow{2}{*}{ On-chip Caches } & $\begin{array}{l}\text { L1 I-cache: 32KB, 4-way, 2-cycle, 1 read port, 1 write port, 64B line size; } \\
\text { L1 D-cache: 32KB, 4-way, 4-bank, 2-cycle, 1 read port, 1 write port, 64B line size; } \\
\text { Unified L2: 512KB (1MB for single core processor), 8-way, 8-bank, 15-cycle, 1 port, 64B line size; }\end{array}$ \\
\hline Prefetcher & Stream prefetcher with 32 streams, prefetch degree of 4, and cache line prefetch distance of 64 (lookahead) [39, 36] \\
\hline
\end{tabular}

TABLE 4

Baseline Shared Resource Configuration

\begin{tabular}{|c|c|}
\hline DRAM controller & $\begin{array}{l}\text { On-chip, demand-first FR-FCFS scheduling policy; } 1 \text { memory controller for 1, 2, 4, 8-core CMP } \\
64,64,128,256 \text {-entry L2 MSHR and memory request buffer for } 1,2,4,8 \text {-core CMP. }\end{array}$ \\
\hline DRAM and bus & $\begin{array}{l}\text { 667MHz DRAM bus cycle, Double Data Rate (DDR3 1333MHz) [18], 16B-wide data bus per memory controller } \\
8 \text { DRAM banks, } 4 \mathrm{~KB} \text { row buffer per bank } \\
\left.\text { Latency: } 15 \mathrm{~ns} \text { per command (precharge }\left({ }^{t} R P\right) \text {, activate }\left({ }^{t} R C D\right) \text {, read/write }(C L)\right), \mathrm{BL}=4 ; \mathrm{AL}=3\end{array}$ \\
\hline
\end{tabular}

$$
\begin{aligned}
A C C= & \frac{\text { Number of useful prefetches }}{\text { Number of prefetches sent }}, \\
C O V= & \{\text { Number of useful prefetches }\} / \\
& \{\text { Number of demand requests } \\
& + \text { Number of useful prefetches. }\}
\end{aligned}
$$

To evaluate the effect of DRAM throughput improvement on the processing core, we define instruction window Stall cycles Per Load instruction (SPL) which indicates, on average, how much time the processor spends idly waiting for DRAM service:

$$
S P L=\frac{\text { Total number of window stall cycles }}{\text { Total number of load instructions }} .
$$

To measure CMP system performance, we use Individual Speedup (IS), Weighted Speedup (WS) [34], and Harmonic mean of Speedups (HS) [15]. As shown by Eyerman and Eeckhout [4], WS corresponds to system throughput and HS corresponds to the inverse of job turnaround time. In the equations that follow, $N$ is the number of cores in the CMP system. IPC alone is the IPC measured when an application runs alone on one core in the CMP system (other cores are idle) and $I P C^{\text {together }}$ is the IPC measured when an application runs on one core, while other applications are running on the other cores of the CMP. Unless otherwise mentioned, we use the demand-first policy to measure $I P C^{\text {alone }}$ for all of our experiments to show the effectiveness of our mechanism on CMP systems:

$$
I S_{i}=\frac{I P C_{i}^{\text {together }}}{I P C_{i}^{\text {alone }}}, W S=\sum_{i}^{N} \frac{I P C_{i}^{\text {together }}}{I P C_{i}^{\text {alone }}}, H S=\frac{N}{\sum_{i}^{N} \frac{I P C_{i}^{\text {alone }}}{I P C_{i}^{\text {together }}}} .
$$

\subsection{Processor Model and Workloads}

We use an in-house cycle accurate x86 CMP simulator for our evaluation. Our processor faithfully models port contention, queuing effects, bank conflicts, and other DDR3 DRAM system constraints. The baseline configuration of each processing core is shown in Table 3 . The shared resource configuration for single, two, four, and eight-core CMPs is shown in Table 4. Note that we evaluate our mechanism on CMP systems with private on-chip last-level
(L2) caches (512 KB for each core) rather than a shared cache to easily show and analyze the effect of PADC in the shared DRAM system by isolating the effect of contention in the DRAM system from the effect of interference in shared caches. However, we evaluate our mechanism for a shared cache in Section 6.9 as well.

We use the SPEC 2000/2006 benchmarks for experimental evaluation. Each single-threaded benchmark was compiled using Intel C Compiler (ICC) or Intel Fortran Compiler (IFORT) with the -O3 option. We ran each benchmark with the reference input set for 200 million $\times 86$ instructions selected by Pinpoints [28] as a representative portion of each benchmark.

We classify the benchmarks into three categories: prefetch-insensitive, prefetch-friendly, and prefetch-unfriendly (class 0,1 , and 2, respectively) based on performance impact a prefetcher has on the application. ${ }^{5}$ The characteristics for a subset of benchmarks with and without a stream prefetcher are shown in Table 5. Only a subset of benchmarks was chosen due to limited space; however, we do evaluate the entire set of 55 benchmarks for single-core experiments for our results. To evaluate our mechanism on CMP systems, we formed combinations of multiprogrammed workloads from the 55 SPEC 2000/2006 benchmarks. We ran 54, 32, and 21 randomly chosen workload combinations (from the 55 SPEC benchmarks) for our two, four, and eight-core CMP configurations, respectively.

For the evaluation of our PADC, we use a prefetch accuracy value of 85 percent for promotion_threshold (for APS) and a dynamic threshold shown in Table 6 for drop_threshold (for APD). The accuracy is calculated every $100 \mathrm{~K}$ cycles.

5. If L2 Misses Per 1K Instructions (MPKIs) increase when the prefetcher is enabled, the benchmark is classified as two. If MPKI without prefetching is greater than 10 and bus traffic increases by more than 75 percent when prefetching is enabled, the benchmark is also classified as two. Otherwise, if IPC increases by five percent, the benchmark is classified as one. Otherwise, it is classified as 0 . Note that memory-intensive applications that experience increased IPC and reduced MPKI (such as milc) may still be classified as prefetch-unfriendly if bus traffic increases significantly. The reason for this is that although an increase in bus traffic may not have much of a performance impact on single-core systems, in CMP systems with shared resources, the additional bus traffic can degrade performance substantially. 
TABLE 5

Characteristics for 28 SPEC 2000/2006 Benchmarks with/without Stream Prefetcher: IPC, L2 Misses Per $1 \mathrm{~K}$ Instructions (MPKIs), RBH, Prefetch Accuracy (ACC), Prefetch Coverage (COV), and Class

\begin{tabular}{|c|c|c|c|c|c|c|c|c|c|c|c|c|c|c|c|c|c|}
\hline & \multicolumn{2}{|c|}{ No prefetcher } & \multicolumn{6}{|c|}{ Prefetcher with demand-first policy } & & \multicolumn{2}{|c|}{ No prefetcher } & \multicolumn{6}{|c|}{ Prefetcher with demand-first policy } \\
\hline Benchmark & IPC & MPKI & IPC & MPKI & $\mathrm{RBH}(\%)$ & $\mathrm{ACC}(\%)$ & $\operatorname{COV}(\%)$ & Class & Benchmark & IPC & MPKI & IPC & MPKI & $\mathrm{RBH}(\%)$ & $\mathrm{ACC}(\%)$ & $\operatorname{COV}(\%)$ & Class \\
\hline eon_00 & 2.08 & 0.01 & 2.08 & 0.00 & 84.93 & 37.37 & 52.64 & $\begin{array}{l}0 \\
\end{array}$ & swim_00 & 0.35 & 27.57 & 0.62 & 8.66 & 42.83 & 99.95 & 68.58 & 1 \\
\hline mgrid_00 & 0.65 & 6.50 & 0.85 & 0.30 & 59.56 & 97.46 & 95.37 & 1 & galgel_00 & 1.42 & 4.26 & 1.10 & 7.56 & 65.50 & 30.96 & 23.94 & 2 \\
\hline art_00 & 0.18 & 89.39 & 0.18 & 65.52 & 91.46 & 35.88 & 34.00 & 2 & equake_00 & 0.42 & 19.87 & 1.00 & 0.76 & 85.02 & 95.63 & 96.19 & 1 \\
\hline facerec_00 & 1.40 & 3.45 & 1.64 & 1.18 & 92.42 & 55.15 & 67.04 & 1 & ammp_00 & 1.70 & 0.80 & 1.47 & 1.70 & 56.20 & 5.96 & 8.03 & 2 \\
\hline lucas_00 & 0.48 & 10.61 & 0.79 & 1.42 & 44.06 & 86.78 & 86.63 & 1 & gcc_06 & 0.55 & 6.28 & 0.81 & 2.23 & 81.57 & 32.62 & 65.37 & 1 \\
\hline mcf_06 & 0.13 & 33.73 & 0.15 & 29.70 & 25.63 & 31.43 & 14.75 & 1 & hmmer_06 & 1.34 & 1.76 & 1.35 & 0.03 & 27.44 & 95.42 & 98.21 & 0 \\
\hline sjeng_06 & 1.57 & 0.38 & 1.57 & 0.38 & 25.13 & 1.67 & 1.11 & 0 & omnetpp_06 & 0.41 & 10.16 & 0.44 & 9.57 & 61.86 & 10.50 & 18.33 & 2 \\
\hline libquantum_06 & 0.41 & 13.51 & 0.65 & 2.75 & 81.39 & 99.98 & 79.63 & 1 & astar_06 & 0.43 & 10.19 & 0.48 & 9.23 & 43.86 & 18.38 & 12.64 & 1 \\
\hline xalancbmk_06 & 0.80 & 1.70 & 0.71 & 2.12 & 49.35 & 8.96 & 13.26 & 2 & bwaves_06 & 0.59 & 18.71 & 1.23 & 0.37 & 83.99 & 99.97 & 98.00 & 1 \\
\hline gamess_06 & 2.11 & 0.04 & 2.13 & 0.01 & 83.97 & 57.80 & 74.73 & 0 & milc_06 & 0.41 & 29.33 & 0.46 & 20.88 & 81.13 & 19.45 & 28.81 & 2 \\
\hline zeusmp_06 & 0.75 & 4.55 & 0.86 & 2.32 & 46.91 & 55.93 & 50.45 & 1 & cactusADM_06 & 0.71 & 4.54 & 0.84 & 2.21 & 33.56 & 45.12 & 51.47 & 1 \\
\hline leslie3d_06 & 0.53 & 20.89 & 0.86 & 2.41 & 77.32 & 89.72 & 88.66 & 1 & soplex_06 & 0.35 & 21.25 & 0.72 & 3.61 & 78.81 & 80.12 & 83.08 & 1 \\
\hline GemsFDTD_06 & 0.44 & 15.61 & 0.80 & 2.02 & 55.82 & 90.71 & 87.12 & 1 & lbm_06 & 0.46 & 20.16 & 0.70 & 2.93 & 58.24 & 94.27 & 85.45 & 1 \\
\hline wrf_06 & 0.62 & 8.10 & 1.03 & 0.60 & 67.14 & 95.23 & 92.63 & 1 & sphinx3_06 & 0.36 & 12.94 & 0.64 & 2.24 & 83.94 & 54.91 & 82.96 & 1 \\
\hline
\end{tabular}

\section{EXPerimental Evaluation}

We first evaluate PADC on single, two, four, and eight-core systems. Section 6.5 analyzes PADC's fairness and proposes new techniques to improve CMP system fairness. Sections 6.6-6.14 analyze the effect of PADC on systems with different configurations and characteristics such as multiple memory controllers, different row buffer policies, different types of prefetchers, prefetch filtering, and runahead execution. This analysis shows that PADC is a general mechanism that is effective for a variety of systems and it is orthogonal to previously proposed prefetching and prefetch filtering techniques.

\subsection{Single-Core Results}

Fig. 6 shows performance of PADC on a single-core system. IPC is normalized to the baseline which employs the demand-first scheduling policy. We show performance of only 15 individual benchmarks due to limited space. The rightmost bars show the average performance of all 55 SPEC CPU 2000/2006 benchmarks (gmean55). As discussed earlier, neither of the rigid scheduling policies (demandfirst, demand-prefetch-equal) provides the best performance across all applications. Demand-first performs better for most prefetch-unfriendly benchmarks (class 2) such as galgel, art, and ammp, while demand-prefetch-equal does better for most prefetch-friendly ones (class 1) such as swim, libquantum, and $\mathrm{lbm}$. Averaged over all 55 SPEC benchmarks, the demand-prefetch-equal policy outperforms demand-first by 0.5 percent since there are more benchmarks (29 out of 55) that belong to class 1 .

APS, shown in the fourth bar from the left, effectively adapts to the behavior of the prefetcher. In most benchmarks, APS provides at least as good performance as the best rigid prefetch scheduling policy. As a result, APS improves performance by 3.6 percent over all 55 benchmarks compared to the baseline. APS (and demand-prefetch-equal) improves

TABLE 6

Dynamic drop_threshold Values for Adaptive Prefetch Dropping Based on Prefetch Accuracy

\begin{tabular}{l||c|c|c|c|c|} 
Prefetch accuracy (\%) & $0-10$ & $10-30$ & $30-70$ & $70-100$
\end{tabular} \begin{tabular}{|l||l|l|l|l|}
\hline drop_threshold (processor cycles) & 100 & 1,500 & 50,000 & 100,000 \\
\hline
\end{tabular} performance over demand-first for many prefetch-friendly applications such as libquantum, bwaves, and leslie3d. This is due to two reasons. First, APS increases DRAM throughput in these applications because it treats demands and prefetches equally most of the time. Doing so improves the timeliness of the prefetcher because prefetch requests do not get delayed behind demand requests. Second, improved DRAM throughput reduces the probability of the memory request buffer being full. As a result, more prefetches are able to enter the request buffer. This improves the coverage of the prefetcher as more useful prefetch requests get a chance to be issued. For example, APS improves the prefetch coverage from 80, 98, and 89 percent to 100, 100, and 92 percent for libquantum, bwaves, and leslie3d, respectively, as shown in Fig. 8.

On the other hand, even though APS is able to provide performance of the best rigid prefetch scheduling policy for each application, it is unable to overcome performance loss due to prefetching in some prefetch-unfriendly applications such as galgel, ammp, and xalancbmk. The prefetcher generates many useless prefetches in these benchmarks that a simple DRAM scheduling policy cannot eliminate.

When APD is employed with demand-first (APD-only), it improves performance for prefetch-unfriendly applications by eliminating many useless prefetches. This is also true when APD is employed with APS (i.e., PADC). Using APD recovers part of performance loss due to prefetching in galgel, ammp, and xalancbmk because it eliminates 54,76, and 54 percent of the useless prefetch requests, respectively, as shown in Fig. 8. As a result, using both of our proposed

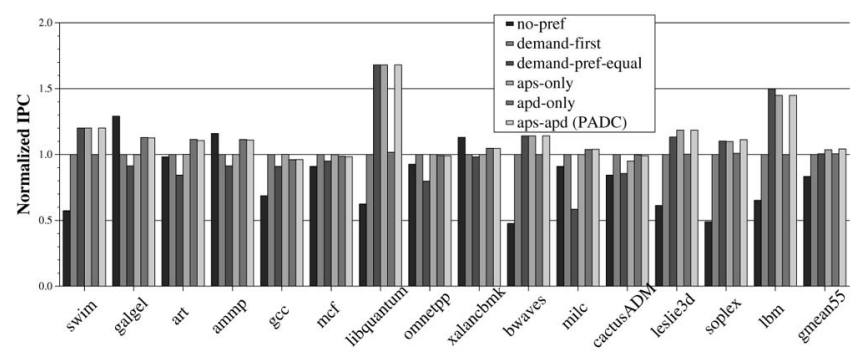

Fig. 6. Performance for 55 SPEC benchmarks on single-core system: Normalized IPC for 15 benchmarks and average for all 55 (gmean55). 


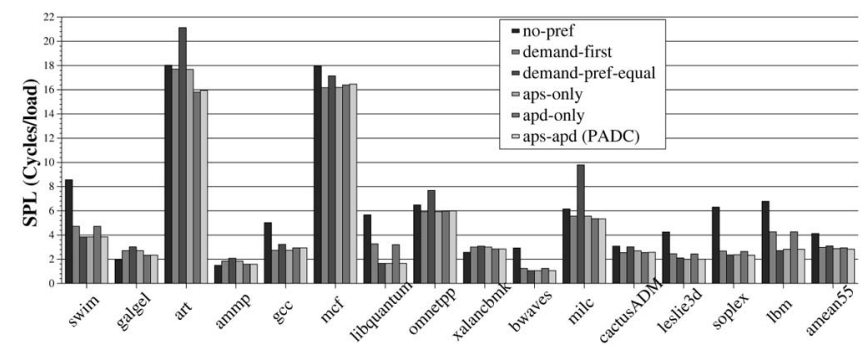

Fig. 7. Stall time per load (SPL) on the single-core system.

mechanisms (APD in conjunction with APS) provides 4.3 percent performance improvement over the baseline.

Fig. 7 provides insight into performance improvement of the proposed mechanisms by showing the effect of each mechanism on the stall time experienced per load instruction (SPL). Our PADC reduces SPL by 5.0 percent compared to the baseline. By providing better DRAM scheduling and eliminating useless prefetches, PADC reduces the amount of time the processor stalls for each load instruction and allows the processor to make faster progress. As a result, PADC significantly improves performance.

Fig. 8 breaks down the bus traffic into three categories: useful prefetches, useless prefetches, and demand requests. PADC reduces bus traffic by 10.4 percent across all benchmarks (amean55) as shown. Reduction in bus traffic is mainly due to APD which significantly reduces the number of useless prefetches. For many benchmarks, APS by itself provides the same bandwidth consumption provided by the best rigid policy for each benchmark. We conclude that our prefetch-aware DRAM controller is very effective at improving both performance and bandwidth efficiency in single-core systems.

Note that simply turning off prefetching for prefetchunfriendly applications may lose opportunity to improve performance. This is true for prefetch-unfriendly applications that have 1) significant phase changes and 2) some accurate prefetches interleaved with inaccurate prefetches. For such benchmarks, prefetching hurts performance in some phases but increases performance significantly in others. If the prefetcher is turned off, performance benefits of useful prefetch phases and useful prefetch requests will be lost. In fact, due to this phase behavior, art and milc do not benefit much from prefetching unless adaptive prefetch management is used. Fig. 6 shows that PADC improves performance significantly for art and milc since it is able to adapt to different phases and eliminate useless prefetches while keeping useful prefetches.

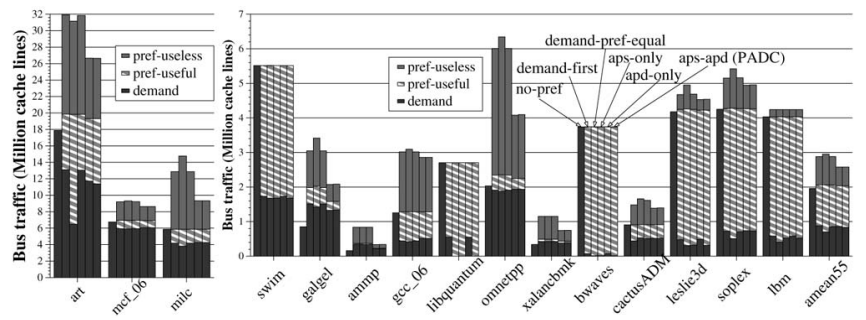

Fig. 8. Bus traffic on the single-core system.

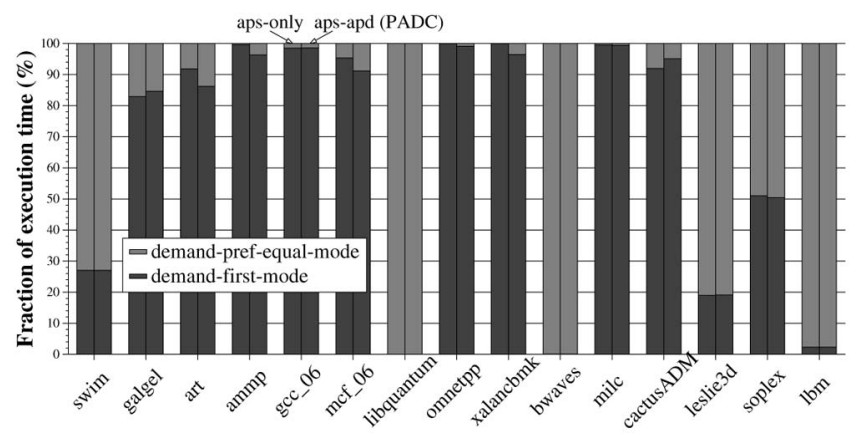

Fig. 9. Fraction of execution time spent in different demand/prefetch scheduling modes on the single-core system.

\subsubsection{Adaptive Behavior of PADC}

We analyze the adaptive runtime behavior of PADC in this section. APS prioritizes demands over prefetches (i.e., demand-first) when the estimated prefetch accuracy is less than promotion_threshold. It treats demands and prefetches equally (i.e., demand-prefetch-equal) when prefetch accuracy is greater than or equal to promotion_threshold. PADC continuously changes the DRAM scheduling mode (between demand-first and demand-prefetch-equal) for the application based on the prefetch accuracy estimated every interval.

Fig. 9 shows the fraction of time APS and PADC spend in each of the two scheduling modes for the single-core system. APS and PADC spend a majority of their execution time in demand-prefetch-equal mode for prefetch-friendly applications but spend most of their execution time in demand-first mode for prefetch-unfriendly applications. Therefore, APS and PADC provide at least as good performance as the best rigid prefetch scheduling policy in most applications, as shown in Fig. 6.

\subsubsection{Effect of PADC on Row Buffer Hit Rate}

Recall that the demand-prefetch-equal policy prioritizes row-hit requests regardless of whether a request is a prefetch or demand. If we consider all demand and prefetch requests (regardless of whether or not a prefetch is useful) for the entire run of an application, the demand-prefetchequal policy will result in the highest row buffer hit rate $(\mathrm{RBH})$, and therefore, the lowest average DRAM access latency among all considered policies. However, this does not mean that this policy performs best since prefetches are NOT always useful as discussed in Section 6.1. When prefetching is enabled, we need a better metric to show how a mechanism reduces effective memory latency. Hereby, we define row buffer hit rate for useful (demand and useful prefetch) requests (RBHU) as follows:

$$
\begin{aligned}
R B H U= & \{\text { Number of row-hit demand requests } \\
& + \text { Number of useful row-hit prefetch requests }\} / \\
& \{\text { Number of demand requests }+ \text { Number of } \\
& \text { useful prefetch requests }\} .
\end{aligned}
$$

The demand-prefetch-equal policy will still show the highest RBHU since RBHU is also maximized by prioritizing row-hit requests. However, a good DRAM scheduling mechanism should keep its RBHU close to demandprefetch-equal's RBHU because it should aim to maximize 
TABLE 7

Row Buffer Hit Rate for Useful (Demand and Useful Prefetch) Requests

\begin{tabular}{|c||c|c|c|c|c|c|c|c|c|c|c|c|c|c|c|}
\hline & swim & galgel & art & ammp & mcf_06 & libquantum & omnetpp & xalancbmk & bwaves & milc & leslie3d & soplex & lbm & amean55 \\
\hline \hline no-pref & 0.18 & 0.51 & 0.94 & 0.40 & 0.12 & 0.86 & 0.47 & 0.23 & 0.76 & 0.85 & 0.71 & 0.81 & 0.53 & 0.55 \\
\hline demand-first & 0.44 & 0.58 & 0.94 & 0.48 & 0.19 & 0.86 & 0.56 & 0.27 & 0.87 & 0.88 & 0.81 & 0.87 & 0.64 & 0.63 \\
\hline demand-pref-equal & 0.50 & 0.58 & 0.96 & 0.50 & 0.23 & 0.98 & 0.59 & 0.28 & 0.89 & 0.90 & 0.91 & 0.93 & 0.92 & 0.68 \\
\hline aps & 0.50 & 0.58 & 0.94 & 0.48 & 0.19 & 0.98 & 0.56 & 0.27 & 0.89 & 0.88 & 0.90 & 0.91 & 0.90 & 0.66 \\
\hline aps-apd (PADC) & 0.50 & 0.56 & 0.94 & 0.44 & 0.18 & 0.98 & 0.54 & 0.25 & 0.89 & 0.88 & 0.90 & 0.90 & 0.90 & 0.65 \\
\hline
\end{tabular}

DRAM bandwidth for useful requests. Table 7 shows RBHU values for 13 benchmarks on the single-core processor with no prefetching, demand-first, demand-prefetch-equal, APS, and PADC. The RBHU of APS is very close to that of demand-prefetch-equal and significantly better than the RBHU of demand-first since APS successfully exploits row buffer locality for useful requests.

Employing APD with APS (i.e., PADC) slightly reduces RBHU for some applications such as galgel, ammp, mcf, omnetpp, xalancbmk, and soplex. This is because adaptive prefetch dropping cancels some useful prefetches as shown in Fig. 8, thereby reducing the fraction of useful row buffer hits. Nonetheless, APD improves overall performance for these applications since it reduces the contention between demands and prefetches by eliminating a significant number of useless prefetches, as discussed in Section 6.1.

\subsection{Two-Core Results}

We briefly discuss only the average performance and bus traffic for the 54 workloads on the two-core system due to space limitations (our main analysis focuses on four-core systems). Fig. 10 shows that PADC improves both performance metrics (WS and HS) by 8.4 and 6.4 percent, respectively, compared to the demand-first policy and also reduces memory bus traffic by 10.0 percent. Thus, the proposed mechanism is effective for dual-core systems. We do not discuss these results further since dual-core processors are no longer the state of the art in multicore systems.

\subsection{Four-Core Results}

We ran 32 different workloads to evaluate the effectiveness of PADC on the four-core system. In the following sections, we discuss three cases in detail to provide insights into the behavior of the Prefetch-Aware DRAM Controller.

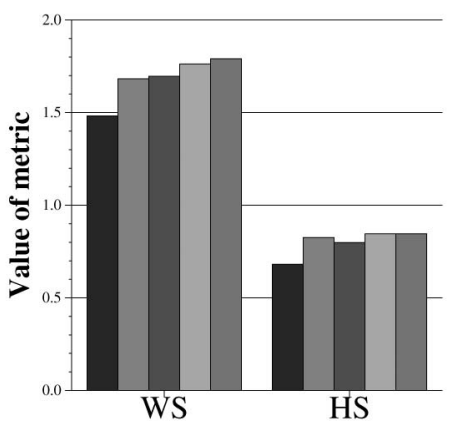

(a)

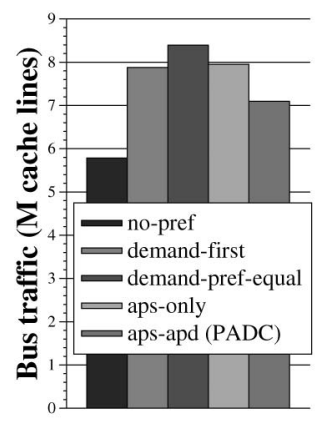

(b)
Fig. 10. Overall performance for 54 workloads on the two-core system. (a) Average system performance. (b) Average traffic.

\subsubsection{Case Study l: All Prefetch-Friendly Applications}

Our first case study examines the behavior of our proposed mechanisms when four prefetch-friendly applications (swim, bwaves, leslie3d, and soplex) run together on the four-core system. Fig. 11a shows the speedup of each application and Fig. 11b shows the system performance.

In addition, Fig. 12 provides insight into performance changes by showing how each mechanism affects stall time per load as well as memory bus traffic. Several observations are in order:

First, since all four applications show very high prefetch coverage (i.e., prefetch-friendly), as shown in Fig. 12b, prefetching provides significant performance improvement in all applications regardless of the DRAM scheduling policy. In addition, the demand-prefetch-equal policy significantly outperforms the demand-first policy (by 28 percent in terms of weighted speedup) because prefetches are very accurate in all four applications. The demand-prefetch-equal policy reduces stall time per load, as shown in Fig. 12a, because it improves DRAM throughput.

Second, our PADC outperforms both of the rigid prefetch scheduling policies improving weighted speedup by 31.3 percent over the baseline demand-first policy. This is because it 1) successfully prioritizes critical (useful) requests over others thereby reducing SPL and 2) drops useless prefetches in leslie3d and soplex thereby reducing their negative effects on all applications. Consequently, our PADC also improves prefetch coverage from 56 to 73 percent, as shown in Fig. 12c. This is because it improves DRAM throughput and reduces contention for memory system resources by dropping useless prefetches from leslie3d and soplex, allowing more useful prefetches to enter the memory system.

Finally, the bandwidth savings provided by PADC is relatively small ( 0.9 percent) because these applications do not generate a large number of useless prefetch requests. However, there is still a non-negligible reduction in bus traffic due to the effective dropping of some useless

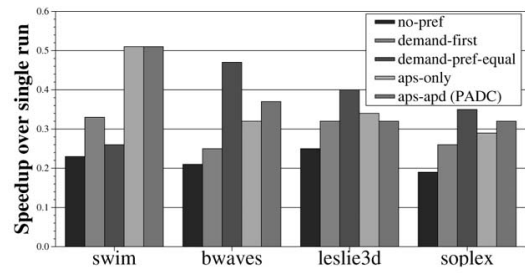

(a)

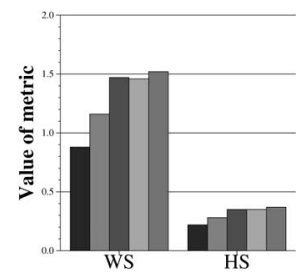

(b)
Fig. 11. A prefetch-friendly four-core workload. (a) Individual application speedup. (b) System performance. 


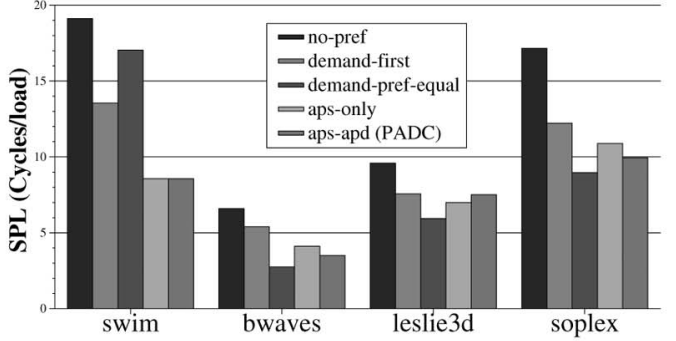

(a)

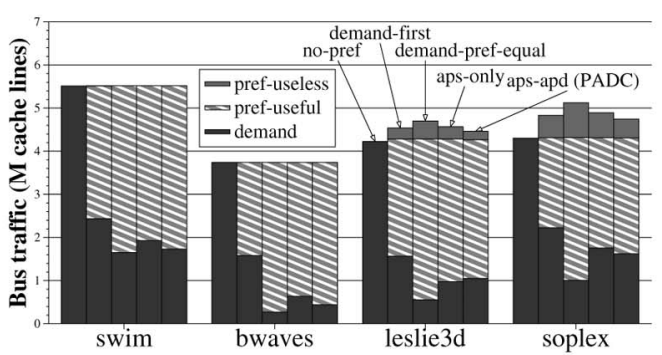

(b)

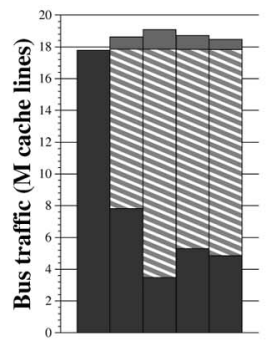

(c)

Fig. 12. A prefetch-friendly four-core workload. (a) SPL. (b) Bus traffic per application. (c) Total bus traffic.

prefetches in leslie3d and soplex. We conclude that the Prefetch-Aware DRAM Controller can provide performance and bandwidth efficiency improvements even when all applications benefit significantly from prefetching.

\subsubsection{Case Study II: All Prefetch-Unfriendly Applications}

Our second-case study examines the behavior of our proposed mechanisms when four prefetch-unfriendly applications (art, galgel, ammp, and milc) run together on the four-core system. Since the prefetcher is very inaccurate for all applications, prefetching degrades performance regardless of the scheduling policy. However, as shown in Fig. 13, the demand-first policy and APS provide better performance than the demand-prefetch-equal policy by prioritizing demand requests over prefetch requests which are more likely to be useless. Employing adaptive prefetch dropping drastically reduces the useless prefetches in all four applications, as shown in Fig. 14b, and therefore, frees up memory system resources to be used by demands and useful prefetch requests. The effect of this can be seen by the reduced SPL as shown in Fig. 14a for all applications. As a result, our PADC performs better than either rigid prefetch scheduling policy for all applications.

PADC improves system performance by 17.7 percent (weighted speedup) and 21.5 percent (harmonic mean of speedups), while reducing bandwidth consumption by 9.1 percent over the baseline demand-first scheduler, as shown in Fig. 14c. By largely reducing the negative effects of useless prefetches both in scheduling and memory system buffers/resources, PADC almost eliminates the system performance loss observed in this prefetch-unfriendly mix of applications. Weighted speedup is within two percent and harmonic mean of speedups is within one percent of those obtained with no prefetching. We conclude that the PrefetchAware DRAM Controller can effectively eliminate the negative performance impact caused by inaccurate prefetching by intelligently managing the scheduling and buffer

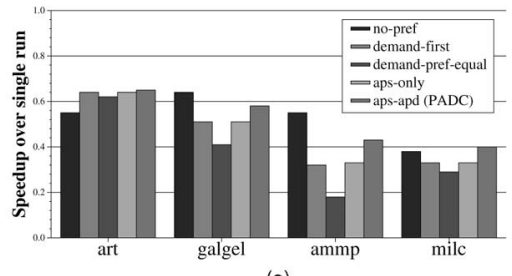

(a)

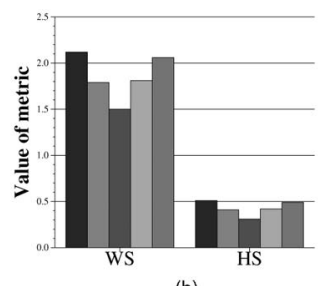

(b)
Fig. 13. A prefetch-unfriendly four-core workload. (a) Individual application speedup. (b) System performance. management of prefetch requests even in workload mixes where prefetching performs inefficiently for all applications.

\subsubsection{Case Study III: Mix of Prefetch-Friendly and Prefetch-Unfriendly Applications}

Figs. 15 and 16 show performance and bus traffic when two prefetch-friendly (libquantum and GemsFDTD) and two prefetch-unfriendly (omnetpp and galgel) applications run together. The prefetches for libquantum and GemsFDTD are very beneficial. Therefore, demand-prefetch-equal significantly improves weighted speedup. However, the prefetcher generates many useless prefetches for omnetpp and galgel, as shown in Fig. 16b. These useless prefetches temporarily deny service to critical requests from the two other cores. Because APD eliminates a large portion (67 and 57 percent) of all useless prefetches in omnetpp and galgel, it frees up both request buffer entries and bandwidth in the memory system. These freed up resources are utilized efficiently by the critical requests of libquantum and GemsFDTD, thereby significantly improving their individual performance while slightly reducing omnetpp and galgel's individual performance. Since it eliminates a large number of useless prefetches, PADC reduces total bandwidth consumption by 14.5 percent over the baseline demand-first policy. We conclude that PADC can effectively prevent the denial of service caused by the useless prefetches of prefetch-unfriendly applications on the useful requests of other applications.

\subsubsection{Effect of Prioritizing Urgent Requests}

In this section, we discuss the effectiveness of prioritizing urgent requests using the application mix in case study III. We say that a multicore system is fair if each application experiences the same individual speedup when multiple applications run together on the system. To indicate the degree of unfairness, we define Unfairness (UF) [4] as follows:

$$
U F=\frac{M A X\left(I S_{0}, I S_{1}, \ldots, I S_{n-1}\right)}{M I N\left(I S_{0}, I S_{1}, \ldots, I S_{n-1}\right)}, N: \text { Number of Cores. }
$$

Table 8 shows individual speedup, unfairness, weighted speedup, and harmonic mean of speedups for the workload from case study III for five policies: demand-first, versions of APS and PADC that do not use the concept of "urgent requests," and regular APS and PADC (with "urgent requests"). If the concept of "urgent requests" is not used, demand requests from the prefetch-unfriendly applications (omnetpp and galgel) unfairly starve because a large number of critical requests from the prefetch-friendly applications (libquantum and GemsFDTD) are given the same priority as those demand requests. This starvation, combined with the 


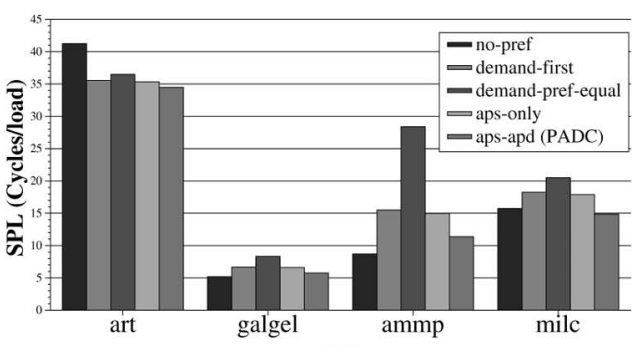

(a)

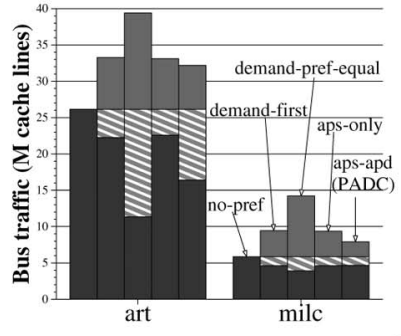

(b)

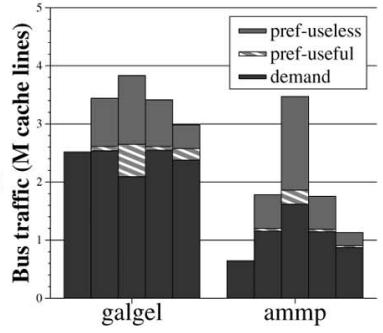

(b)

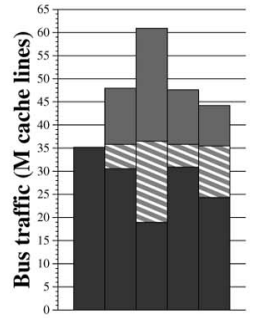

(c)

Fig. 14. A prefetch-unfriendly four-core workload. (a) SPL. (b) Bus traffic per application. (c) Total bus traffic.

negative effects of useless prefetches, leads to unacceptably low individual speedups for these applications resulting in high unfairness. When urgency is used to prioritize requests, this unfairness is significantly mitigated, as shown in Table 8. In addition, harmonic mean of speedups (i.e., average job turnaround time) significantly improves at the cost of very little weighted speedup (i.e., system throughput) degradation. However, we found that for most workloads (30 out of the 32), prioritizing urgent requests improves weighted speedup as well. This trend holds true for most workload mixes that consist of prefetch-friendly and prefetch-unfriendly applications. On average (not shown in the table), prioritizing urgent requests improves UF, HS, and WS by 13.7, 8.8, and 3.8 percent, respectively, compared to PADC with no concept of urgency for the 32 four-core workloads. We conclude that incorporating the concept of urgency into PADC significantly improves system fairness while keeping system performance high.

\subsubsection{Effect on Identical Application Workloads}

It is common that commercial servers frequently run multiple instances of identical applications. In this section, we evaluate the effectiveness of PADC when the four-core system runs four identical applications together. Since APS prioritizes memory requests and APD drops useless prefetches (both based on the estimated prefetch accuracy), PADC should evenly improve individual speedup of each instance of the identical applications running together. In other words, all instances of the application are likely to show the same behavior and the same adaptive decision should be made for every interval.

Table 9 shows the system performance of PADC when four instances of libquantum run together on the four-core system. Because libquantum is very prefetch-friendly and most prefetches are row-hits, the demand-prefetch-equal policy performs very well by achieving almost the same speedup for all four instances. APS and PADC perform similarly to demand-prefetch-equal (improving weighted

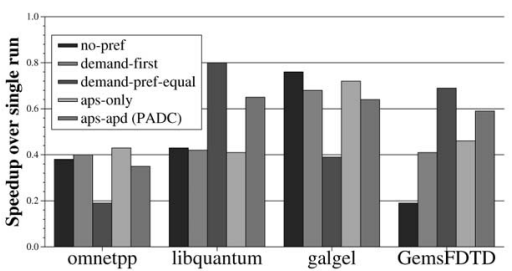

(a)

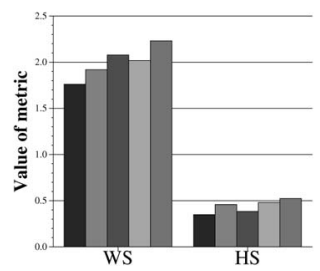

(b)
Fig. 15. A mixed four-core workload. (a) Individual application speedup. (b) System performance. speedup by 18.2 percent compared to demand-first) since they successfully treat demands and prefetches equally for all four instances.

Table 10 shows the system performance of PADC when four instances of a prefetch-unfriendly application, milc, run together on the four-core system. Because the prefetches generated for each instance are useless for most of the execution time of milc, demand-first and APS outperform demand-prefetch-equal for each instance. Incorporating APD into APS (i.e., PADC) further improves individual speedup of all instances equally by reducing useless prefetches from each instance. As a result, PADC significantly improves all system performance metrics. In fact, using PADC allows the system to gain significant performance improvement from prefetching, whereas using a rigid prefetch scheduling policy results in a large performance loss due to prefetching. To conclude, PADC is also very effective when multiple identical applications run together on a CMP system.

\subsubsection{Overall Performance}

Fig. 17 shows the average system performance and bus traffic for the 32 workloads run on the four-core system. PADC provides the best performance and lowest bandwidth consumption compared to all previous prefetch handling policies. It improves weighted speedup and harmonic mean of speedups by 8.2 and 4.1 percent, respectively, compared to the demand-first policy, and reduces bus traffic by 10.1 percent over demand-first (the best performing rigid policy).

We found that PADC outperforms both the demand-first and demand-prefetch-equal policies for all but one workload we examined. The worst performing workload is the combination of vpr, gamess, dealII, and calculix. PADC's WS degradation is only 1.2 percent compared to the demandfirst policy. These applications are either insensitive to prefetching (class 0 ) or not memory-intensive (vpr).

\subsection{Eight-Core Results}

Fig. 18 shows the average performance and bus traffic over the 21 workloads we simulated on the eight-core system. Note that the rigid prefetch scheduling policies actually cause stream prefetching to degrade performance in the eight-core system. The demand-first policy reduces performance by 1.2 percent and the demand-prefetch-equal policy by 3.0 percent compared to no prefetching. DRAM bandwidth becomes a lot more valuable with the increased number of cores because the cores put more pressure on the memory system. At any given time, there are a much larger number of demand and useful/useless prefetch requests in the memory request buffer. As a result, it becomes more likely that 1) a useless prefetch delays a demand or useful prefetch (if 


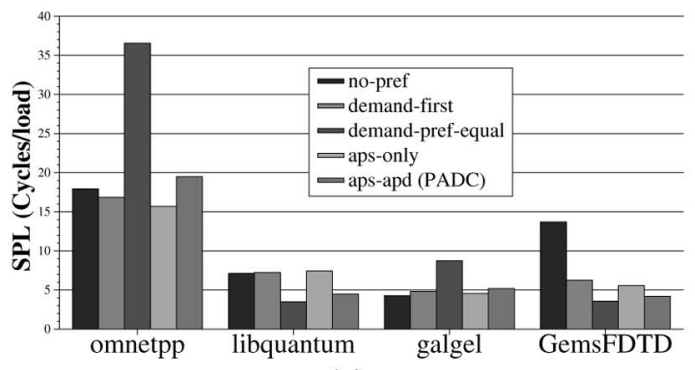

(a)

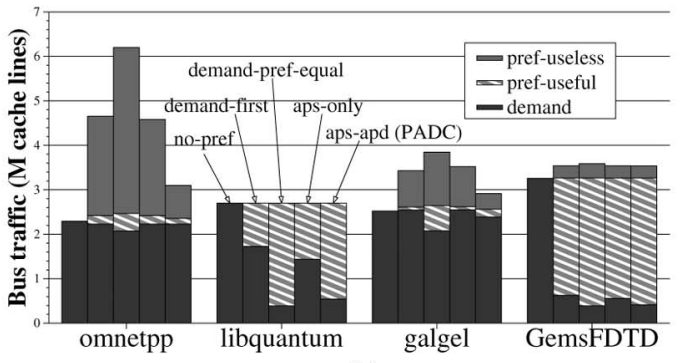

(b)

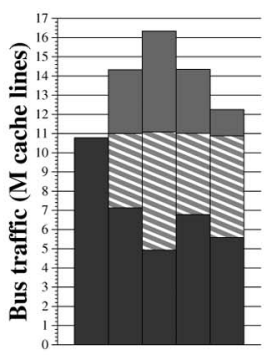

(c)

Fig. 16. A mixed four-core workload. (a) SPL. (b) Bus traffic per application. (c) Total bus traffic.

demand-prefetch-equal policy is used) and 2) DRAM throughput degrades if a demand request causes significant reduction in the row buffer locality of prefetch requests (if demand-first policy is used). Hence, performance degrades with a rigid scheduling policy.

For the very same reasons, PADC becomes more effective when the number of cores increases. As resource contention becomes higher, performance benefit of intelligent prioritization and dropping of useless prefetch requests increase. Our PADC improves overall system performance (WS) by 9.9 percent and also reduces memory bandwidth consumption by 9.4 percent on the eight-core system. We conclude that the benefits of PADC will continue to increase as off-chip memory bandwidth becomes a bigger performance bottleneck in future systems with many cores.

TABLE 8

Effect of Prioritizing Urgent Requests

\begin{tabular}{|c||c|c|c|c||c|c|c|}
\hline \multicolumn{1}{|c||}{} & \multicolumn{4}{c||}{ Individual speedup } & \multirow{2}{*}{ UF } & WS & HS \\
\cline { 2 - 6 } & omnetpp & libquantum & galgel & GemsFDTD & & & \\
\hline \hline demand-first & 0.40 & 0.42 & 0.68 & 0.41 & 1.69 & 1.92 & 0.46 \\
\hline aps-no-urgent & 0.26 & 0.68 & 0.47 & 0.61 & 2.57 & 2.02 & 0.44 \\
\hline aps & 0.43 & 0.41 & 0.72 & 0.46 & 1.73 & 2.02 & 0.48 \\
\hline aps-apd-no-urgent & 0.21 & 0.94 & 0.42 & 0.70 & 4.55 & 2.26 & 0.41 \\
\hline aps-apd (PADC) & 0.35 & 0.65 & 0.64 & 0.59 & 1.84 & 2.23 & 0.52 \\
\hline
\end{tabular}

TABLE 9

Effect on Four Identical Prefetch-Friendly (libquantum) Applications on Four-Core System

\begin{tabular}{|c|c|c|c|c|c|c|c|}
\hline & \multicolumn{4}{|c|}{ Individual speedup } & \multirow{2}{*}{ WS } & \multirow{2}{*}{ HS } & \multirow{2}{*}{ UF } \\
\hline & libquantum & libquantum & libquantum & libquantum & & & \\
\hline no-pref & (20.60 & "0.60 & (20.60 & "0.59 & 2.40 & 0.60 & 1.01 \\
\hline demand-first & 0.69 & 0.67 & 0.65 & 0.64 & 2.66 & 0.66 & 1.08 \\
\hline demand-pref-equal & 0.80 & 0.79 & 0.78 & 0.77 & 3.14 & 0.78 & 1.05 \\
\hline aps & 0.80 & 0.79 & 0.78 & 0.77 & 3.14 & 0.79 & 1.04 \\
\hline aps-apd (PADC) & 0.80 & 0.79 & 0.78 & 0.77 & 3.14 & 0.79 & 1.04 \\
\hline
\end{tabular}

TABLE 10

Effect on Four Identical Prefetch-Unfriendly (milc) Applications on Four-Core System

\begin{tabular}{|c||c|c|c|c||c|c|c|}
\hline \multicolumn{1}{|c||}{} & \multicolumn{2}{|c|}{ Individual speedup } & \multirow{2}{*}{ WS } & HS & UF \\
\cline { 2 - 5 } & milc & milc & milc & milc & & & \\
\hline \hline no-pref & 0.53 & 0.53 & 0.53 & 0.53 & 2.11 & 0.53 & 1.00 \\
\hline demand-first & 0.52 & 0.51 & 0.50 & 0.46 & 1.99 & 0.50 & 1.13 \\
\hline demand-pref-equal & 0.36 & 0.36 & 0.36 & 0.36 & 1.45 & 0.36 & 1.01 \\
\hline aps & 0.52 & 0.51 & 0.50 & 0.46 & 1.99 & 0.50 & 1.14 \\
\hline aps-apd (PADC) & 0.59 & 0.58 & 0.58 & 0.58 & 2.33 & 0.58 & 1.02 \\
\hline
\end{tabular}

\subsection{Optimizing PADC for Fairness Improvement in CMP Systems: Incorporating Request Ranking}

PADC can be better tuned and optimized for the requirements of CMP systems. One major issue in designing memory controllers for CMP systems is the need to ensure fair access to memory by different cores [26], [22], [23]. So far, we have considered PADC only as a way to improve overall system performance. However, to be more effective in CMP systems, PADC can be augmented with a mechanism that provides fairness to different cores' requests. To achieve this purpose, this section describes a new scheduling algorithm that incorporates a request ranking scheme similar to the one used in Parallelism-Aware Batch Scheduling (PAR-BS) [23] into our APS mechanism.

Recall that APS prioritizes urgent requests (demand requests from cores whose prefetch accuracy is low) over others to mitigate performance degradation and unfairness for prefetch-unfriendly applications. However, APS follows

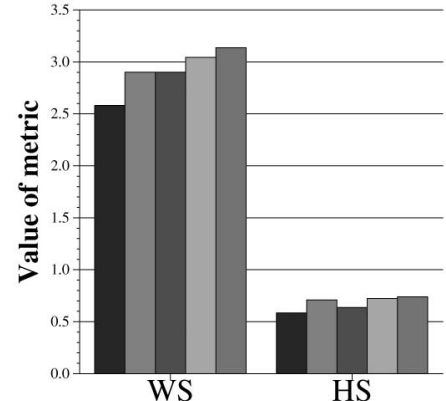

(a)

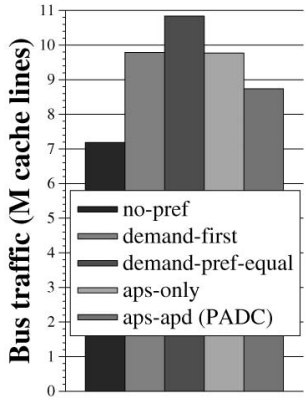

(b)
Fig. 17. Overall performance for 32 workloads on four-core system. (a) Average system performance. (b) Average traffic.

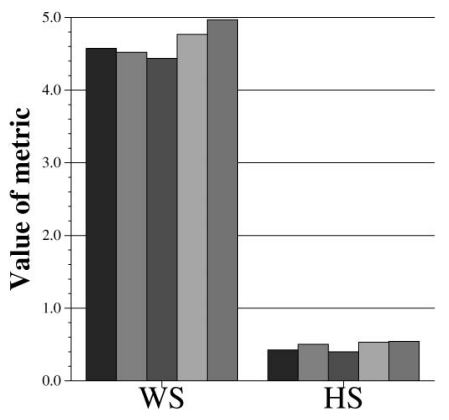

(a)

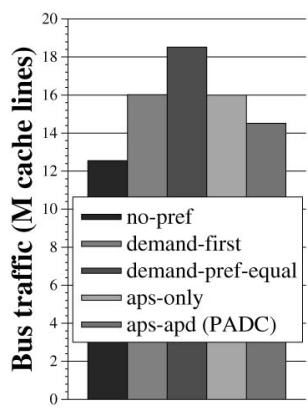

(b)
Fig. 18. Overall performance for 21 workloads on eight-core system. (a) Average system performance. (b) Average traffic. 
the FCFS policy if all other priorities (i.e., criticality, row-hit, and urgency) are the same. This FCFS rule can degrade fairness and system performance by prioritizing requests of memory-intensive applications over those of memory nonintensive applications, as was shown in previous work [26], [22], [23]. This happens because delaying the requests of memory nonintensive applications results in a lower individual speedup (or a higher slowdown) for those applications than it would for memory-intensive applications which already suffer from long DRAM service time. Therefore, PADC (APS) itself cannot completely solve the unfairness problem. This is especially true in cases where all of the applications behave the same in terms of prefetch friendliness (either all are prefetch-friendly or all are prefetch-unfriendly). In such cases, PADC will likely degenerate into the FCFS policy (since the criticality, rowhit, and urgency priorities would be equal) resulting in high unfairness and performance degradation. For example, in case study II discussed in Section 6.3.2, all the applications are prefetch-unfriendly. Therefore, PADC prioritizes demands over prefetches most of the time. PADC mitigates performance degradation by prioritizing demand requests and dropping useless prefetches. However, art is very memory-intensive and continuously generates many demand requests. These demand requests significantly interfere with other applications' demand requests resulting in high slowdowns for the other applications. However, art experiences very little slowdown thereby creating unfairness in the system.

To take into account fairness in PADC, we incorporate the concept of ranking, as employed by Mutlu and Moscibroda [23]. Our ranking scheme is based on the shortest job first principle [33] which can better mitigate the unfairness problem and performance degradation caused by the FCFS rule, as explained in detail by Mutlu and Moscibroda [23]. For each application, the DRAM controller keeps track of the total number of critical (demand and useful prefetch) requests in the memory request buffer. Applications with fewer outstanding critical requests are given a higher rank. The insight is that if an application that has fewer critical requests is delayed, the impact of that delay on that application's slowdown is much higher than the impact of delaying an application with a large number of critical requests. In other words, it is more unfair to delay an application that has a small number of useful requests (i.e., a "shorter" application/job) than delaying an application that has a large number of useful requests (i.e., a "longer" application/job). To achieve this while still being prefetch aware, the DRAM controller schedules memory requests based on the modified rule shown in Rule 2. A highly ranked request is scheduled by the DRAM controller when all requests in the memory request buffer have the same priority for criticality, row-hit, and urgency.

Rule 2. Adaptive prefetch scheduling with ranking

1. Critical request $(C)$ : Critical requests are prioritized over all other requests.

2. Row-hit request (RH): Row-hit requests are prioritized over row-conflict requests.

3. Urgent request (U): Demand requests generated by cores with low prefetch accuracy are prioritized over other requests.

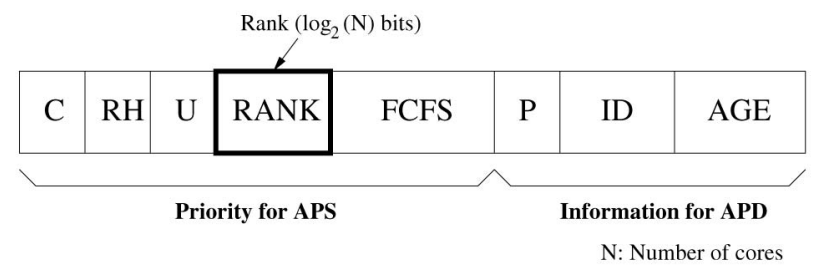

Fig. 19. Memory request fields for PADC with ranking.

4. Highest rank request (RANK): Critical requests from a higher ranked core are prioritized over critical requests from a lower ranked core. Critical requests from cores that have fewer outstanding critical requests are ranked higher.

5. Oldest request (FCFS): Older requests are prioritized over younger requests.

To implement ranking, the priority field for each memory request is augmented, as shown in Fig. 19. A counter per core is required to keep track of the total number of critical requests in the memory request buffer. When the estimated prefetch accuracy of a core is greater than promotion_threshold, the total number of outstanding demand and prefetch requests (critical requests) for that core is counted. When the accuracy is less than the threshold, the counter stores only the number of outstanding demand requests. Cores are ranked according to the total number of critical requests they have in the memory request buffer: a core that has a higher number of critical requests is ranked lower. The RANK field of a request is the same as the rank value of the core determined in this manner. As such, the critical requests of a core with a lower value in its counter are prioritized. This process is done every DRAM bus cycle in our implementation. Alternatively, determination of the ranking can be done periodically since it does not need to be highly accurate and is not on the critical path.

Note that in this study, we do not rank noncritical requests (i.e., prefetch requests from cores whose prefetch accuracy is low). The RANK field of these requests is automatically set to 0 (the lowest rank value). We evaluated a mechanism that also ranks noncritical requests based on estimated prefetch accuracy, and found that this mechanism does not perform better than the mechanism that ranks only critical requests.

Fig. 20 shows the average system performance, bus traffic, and unfairness when we incorporate the ranking mechanism into PADC for the 32 four-core workloads. On

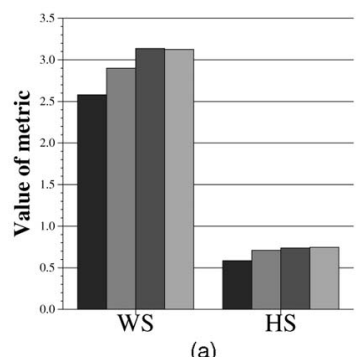

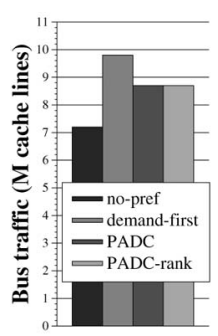

(b)

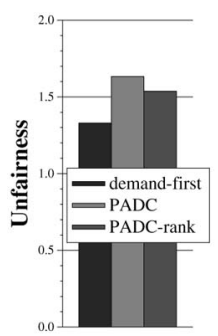

(c)
Fig. 20. Optimized PADC using ranking mechanism on four-core system. (a) Performance. (b) Average traffic. (c) Unfairness. 


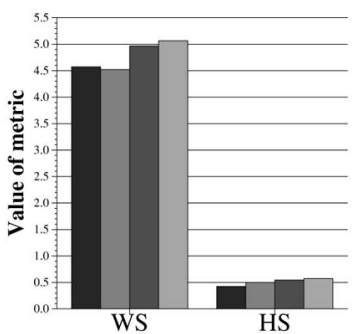

(a) (b)

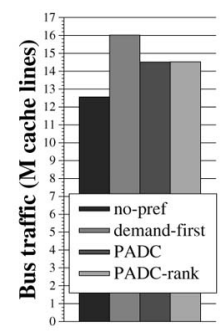

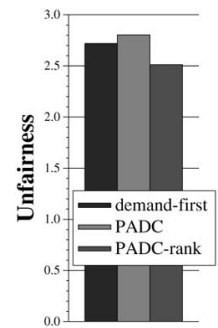

(c)
Fig. 21. Optimized PADC using ranking mechanism on eight-core system. (a) Performance. (b) Average traffic. (c) Unfairness.

average, the ranking mechanism slightly degrades weighted speedup (by 0.4 percent) and slightly improves harmonic mean of speedups (by 0.9 percent) and keeps bandwidth consumption about the same compared to the original PADC. Unfairness is improved from 1.63 to 1.53 . The performance improvement is not significant because the contention in the memory system is not very high in the four-core system. Nonetheless, the ranking scheme improves all the system performance and unfairness metrics for most workloads with memory-intensive benchmarks. For the workload in case study II, the ranking scheme improves WS, HS, and UF by 7.5, 10.3, and 15.1 percent compared to PADC without ranking.

We also evaluate the optimized PADC scheme with ranking on the eight-core system which places significantly more pressure on the DRAM system. As shown in Fig. 21, the ranking mechanism improves WS and HS by 2.0 and 5.4 percent, respectively, and reduces unfairness by 10.4 percent compared to PADC without ranking. The effectiveness of the ranking scheme is much higher in the eight-core system than the four-core system since it is more critical to schedule memory requests fairly in many-core bandwidthlimited systems. Improving fairness reduces starvation of some cores resulting in improved utilization of the cores in the system which, in turn, results in improved system performance. Since starvation is more likely when the memory system is shared between eight cores rather than four, performance improvement obtained with the ranking scheme is higher in the eight-core system.

We conclude that augmenting PADC with an intelligent fairness mechanism improves both unfairness and system performance.

\subsection{Effect on Multiple DRAM Controllers}

We also evaluate performance impact of PADC when two DRAM controllers are employed in the four and eight-core systems. Each memory controller works independently through a dedicated channel (address and data buses) doubling the peak memory bandwidth. Because there is more bandwidth available in the system, contention between prefetch and demand requests is significantly reduced. Therefore, the baseline system performance is significantly improved compared to the single controller. Adding one more DRAM controller improves weighted speedup by 16.9 and 30.9 percent compared to the single controller for four and eight-core systems, respectively.

Figs. 22 and 23 show the average performance and bus traffic for four and eight-core systems with two memory controllers. Note that for the eight-core system, unlike the single memory controller configuration shown

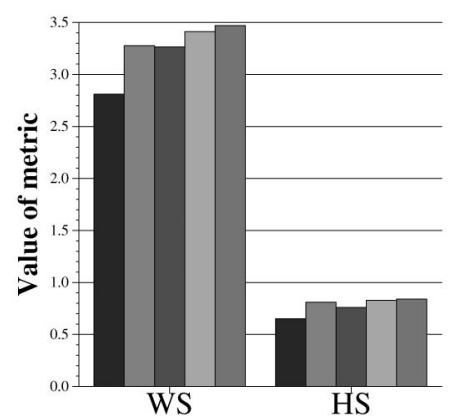

(a)

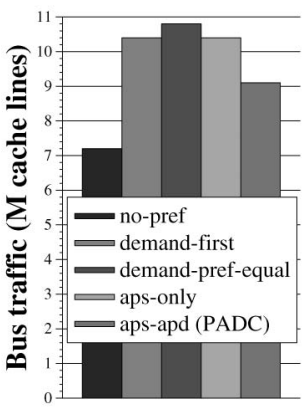

(b)
Fig. 22. Performance of PADC on four-core system with dual-memory controllers. (a) Average system performance. (b) Average traffic.

in Fig. 18a where adding a prefetcher actually degrades performance, performance increases when adding a prefetcher even for the rigid scheduling policies because of the increased memory bandwidth.

PADC is still very effective with two memory controllers and improves weighted speedup by 5.9 and 5.5 percent and also reduces bandwidth consumption by 12.9 and 13.2 percent compared to the demand-first policy for four and eight-core systems, respectively. Therefore, we conclude that PADC still performs effectively on a multicore processor with very high DRAM bandwidth.

\subsection{Effect with Different DRAM Row Buffer Sizes}

As motivated in Section 3, PADC takes advantage of and relies on the row buffer locality of demand and prefetch requests generated at runtime. To determine the sensitivity of PADC to row buffer size, we varied the size of the row buffer from 2 to $128 \mathrm{~KB}$ for the 32 workloads run on the four-core system. Fig. 24 shows the WS improvements of PADC and APS compared to no prefetching, demand-first, and demand-prefetch-equal.

PADC consistently outperforms no prefetching, demand-first, and demand-prefetch-equal with various row buffer sizes. Note that the demand-first policy starts degrading performance compared to no prefetching as the row buffer becomes very large (64 and $128 \mathrm{~KB}$ ). This is because preserving row buffer locality for useful requests is more critical when the row buffer size is large (especially when the stream prefetcher is enabled). No prefetching with larger row buffer sizes exploits row buffer locality

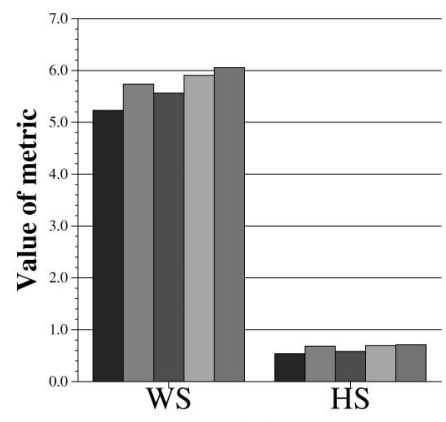

(a)

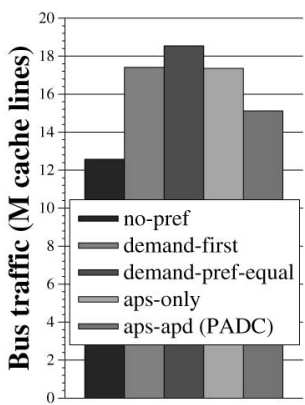

(b)
Fig. 23. Performance of PADC on eight-core system with dual-memory controllers. (a) Average system performance. (b) Average traffic. 


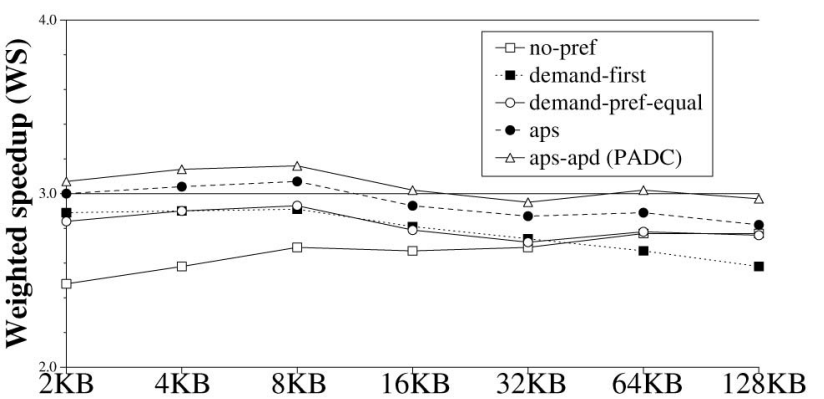

Fig. 24. Effect of PADC with various DRAM row buffer sizes on four-core system.

more (higher row-hit rate) than smaller row buffer sizes. However, with demand-first, the negative performance impact of frequent reactivations of DRAM rows for demand and prefetch requests becomes significantly worse at larger row buffer sizes. Therefore, the demand-first policy experiences a higher memory service time, on average, than no prefetching with large row buffer sizes.

Similarly, the demand-prefetch-equal policy does not improve performance compared to no prefetching for 64 and $128 \mathrm{~KB}$ row buffer sizes since it does not take into account the usefulness of prefetches. With a large row buffer, useless prefetches have higher row buffer locality because many of them hit in the row buffer due to the streaming nature of the prefetcher. As a result, demandprefetch-equal significantly delays the service of demand requests at large row buffer sizes by servicing more useless row-hit prefetches first.

In contrast to these two rigid scheduling policies, PADC tries to service only useful row-hit memory requests first, thereby significantly improving performance even for large row buffer sizes (8.8 and 7.3 percent compared to no prefetching for 64 and $128 \mathrm{~KB}$ row buffers). Therefore, PADC can make a prefetcher viable and effective even when a large row buffer size is used because it takes advantage of the increased row buffer locality opportunity provided by a larger row buffer only for useful requests instead of wasting the increased amount of bandwidth enabled by a larger row buffer on useless prefetch requests.

\subsection{Effect with a Closed-Row DRAM Row Buffer Policy}

So far, we have assumed that the DRAM controller employs the open-row policy (i.e., it keeps the accessed row open in the row buffer after the access even if there are no more outstanding requests requiring the row). In this section, we evaluate the effectiveness of PADC with a closed-row policy. The closed-row policy closes (by issuing a precharge command) the currently-opened row when all row-hit requests in the memory request buffer have been serviced by the DRAM controller. This policy can hide effective precharge time by 1) overlapping the precharge latency with the row-access latency [18] and 2) issuing the precharge command (closing a row buffer) earlier than the open-row policy (thereby converting a row-conflict access into a row-closed access if the next access is to a different row). Therefore, if no more requests to the same row arrive at the memory request buffer after a row buffer

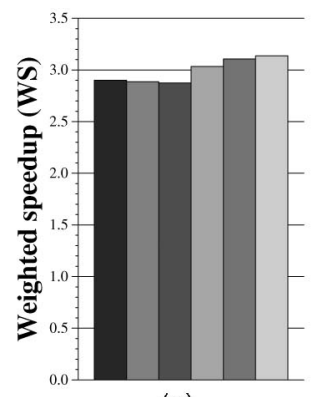

(a)

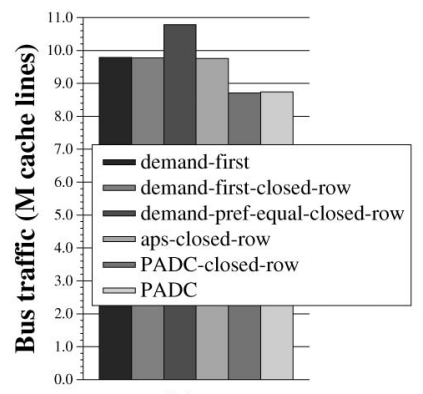

(b)
Fig. 25. Effect on closed-row policy. (a) Average system Performance. (b) Average traffic.

is closed by a precharge command, the closed-row policy can outperform the open-row policy. This is because with the closed-row policy, the later requests do not need a precharge before activating the different row. However, if a request to the same row arrives at the memory request buffer soon after the row is closed, this policy has to pay a penalty (the sum of the nonoverlapped precharge latency and the activation latency) which would not have been required for the open-row policy. Consequently, for applications that have high row buffer locality (i.e., applications that generate bursty row-hit requests) such as streaming/striding applications, the open-row policy outperforms the closed-row policy by reducing reactivations of the same rows that will be needed again in the near future.

Since the closed-row policy still services row-hit requests first until no more requests to the same row remain in the memory request buffer, it can increase DRAM throughput within the scope of the requests that are outstanding in the memory request buffer. When a prefetcher is enabled with the closed-row policy, the same problem exists as for the open-row policy: none of the rigid prefetch scheduling policies can achieve the best performance for all applications since they are not aware of prefetch usefulness. Therefore, PADC can still work effectively with the closedrow policy as we show empirically below.

Fig. 25 shows performance and bus traffic when PADC is used with the closed-row policy for the 32 four-core workloads. The closed-row policy with demand-first scheduling slightly degrades performance by 0.5 percent compared to the open-row policy with demand-first scheduling. This is because there are a large number of streaming/striding (and prefetch-friendly) applications in the SPEC 2000/2006 benchmarks whose performance can be significantly improved with the open-row policy. The performance improvement of the open-row policy is not very significant because there is also a large number of applications that work well with the closed-row policy as they do not have high row buffer locality.

The results show that PADC is still effective with the closed-row policy since it still effectively exploits row buffer locality (within the scope of the requests outstanding in the memory request buffer) and reduces the negative effects of useless prefetch requests. PADC improves weighted speedup by 7.6 percent and reduces bandwidth consumption by 10.9 percent compared to demand-first scheduling with the closed-row policy. Note that PADC with the open-row policy slightly outperforms PADC with the closed row by 


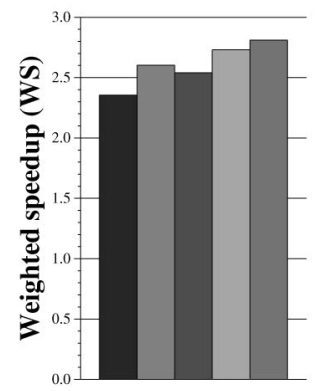

(a)

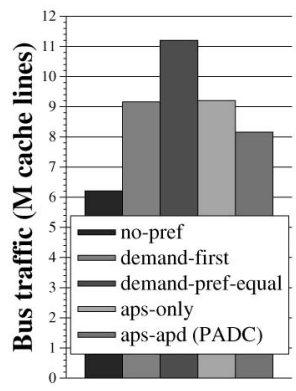

(b)

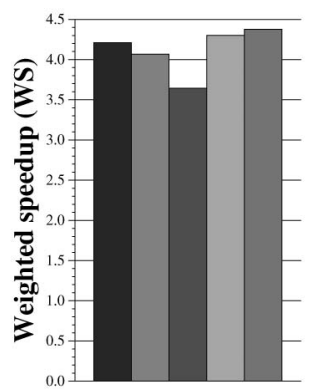

(a)

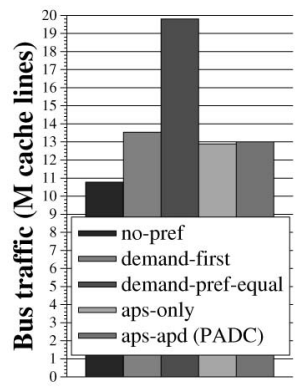

(b)
Fig. 26. Effect on shared L2 cache on four-core system. (a) Performance. (b) Average traffic.

1.1 percent for weighted speedup. Overall, we conclude that PADC is suitable for different row buffer management policies, but it is more effective with the open-row policy due to the existence of a larger number of benchmarks with high row buffer locality.

\subsection{Effect with a Shared Last-Level Cache}

Throughout the paper, we evaluate our mechanism on CMP systems with private on-chip last-level (L2) caches rather than a shared cache where all cores share a large on-chip last-level cache. This allowed us to easily show and analyze the effect of PADC in the shared DRAM system by isolating the effect of contention in the DRAM system from the effect of interference in shared caches. However, many commercial processors already employ shared last-level caches in their CMP designs [39], [40]. In this section, we evaluate performance of PADC in on-chip shared L2 caches on the four and eight-core systems to show the effectiveness of PADC in systems with a shared last-level cache.

For this experiment, we use a shared L2 cache whose size is equivalent to the sum of all the private L2 cache sizes in our baseline system. We scaled the associativity of the shared cache with the number of cores on the chip since as the number of cores increases, the contention for a cache set increases. Therefore, the four-core system employs a 2-MB, 16-way set-associative cache and the eight-core system has a 4-MB, 32-way set-associative cache. We selected 32-way setassociativity for the eight-core system in order to show how the mechanism works with a very aggressive last-level cache. If the associativity is less, our mechanism performs even better. We also assume that each core employs its own independent stream prefetcher that monitors the core's demand accesses and sends prefetched data into the shared L2 cache. However, our mechanism can also work for a single prefetcher which monitors all cores' accesses and generates prefetches for all cores [39], [40] by simply associating core ID bits with each prefetch request, signifying which core generated the prefetch request. This way, PADC can update the appropriate per-core counters to estimate prefetch accuracy of each core.

Figs. 26 and 27 show weighted speedup and average bus traffic on the four and eight-core systems with shared L2 caches. PADC outperforms demand-first by 8.0 and 7.6 percent on the four and eight-core systems, respectively. We conclude that PADC works efficiently for shared lastlevel caches as well.
Fig. 27. Effect on shared L2 cache on eight-core system. (a) Performance. (b) Average traffic.

Note that the demand-prefetch-equal policy does not work well on either of the shared cache systems (degrading WS by 2.4 and 10.4 percent compared to demand-first for four and eight-core systems). This is because the contention in the shared cache among the requests from different cores significantly increases compared to that of a private cache system. With private caches, useless prefetches from one core can only replace useful lines of that same core. However, with a shared cache, useless prefetches from one core can also replace the useful lines of all the other cores. These replaced lines must be brought back into the cache again from DRAM when they are needed. Therefore, the total bandwidth consumption significantly increases. This cache contention among cores becomes especially worse with demandprefetch-equal for prefetch-unfriendly applications. This is because the demand-prefetch-equal policy results in high cache pollution since it blindly prefers to increase DRAM throughput without considering the usefulness of prefetches. The demand-prefetch-equal policy increases bus traffic by 22.3 and 46.3 percent compared to demand-first for the four and eight-core systems, as shown in Figs. 26b and 27b. In contrast, PADC delays the service of useless prefetches and also drops them thereby mitigating contention in both the shared cache and the shared DRAM system.

\subsection{Effect with Different Last-Level Cache Sizes}

PADC aims to maximize DRAM throughput for useful memory (demand and useful prefetch) requests and to delay and drop useless memory requests. One might think that a prefetch/demand management technique such as PADC would not be needed for larger L2 (last-level) caches since a larger cache can reduce cache misses (i.e., memory requests). However, a prefetcher can still generate a significant number of useful prefetch requests for some applications or program phases by correctly predicting demand access patterns which cannot be stored even in large caches due to the large working set size or streaming nature of the program. In addition, the prefetcher can issue a significant number of useless prefetches for other applications or program phases. For these reasons, the interference between demands and prefetches still exists in systems with large caches. Therefore, we hypothesize that PADC is likely to be effective in systems with large last-level caches.

To test this hypothesis, we evaluate the effectiveness of PADC for various L2 cache sizes. We vary the private L2 cache size from $512 \mathrm{~KB}$ to $8 \mathrm{MB}$ per core and the shared 


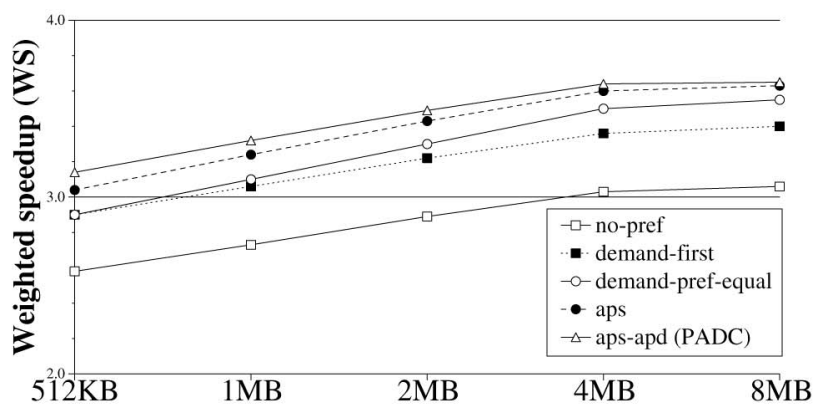

(a)

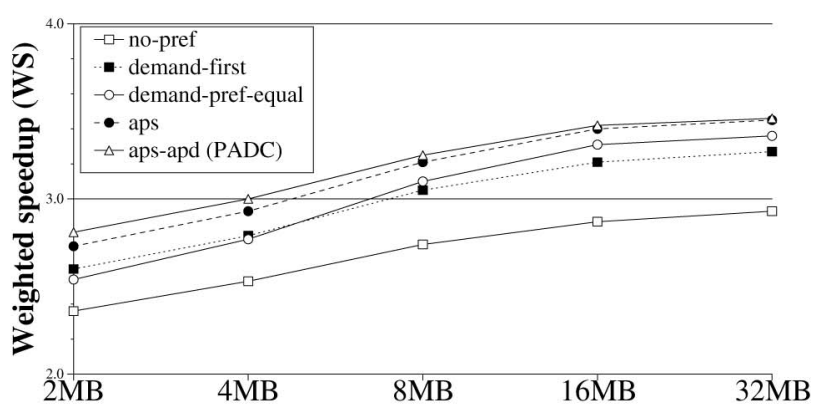

(b)

Fig. 28. Effect of PADC on various cache sizes per core on four-core system. (a) Private L2 caches per core. (b) Shared L2 cache.

cache size from 2 to $32 \mathrm{MB}$ (other cache parameters are as described in Section 6.9) on our four-core CMP system. Fig. 28 shows the system performance (weighted speedup) for the 32 four-core workloads.

As expected, with larger cache sizes, baseline system performance improves. However, the stream prefetcher still effectively improves performance compared to no prefetching with either the demand-first or the demand-prefetchequal policy. In addition, PADC consistently and significantly improves performance compared to both demandfirst and demand-prefetch-equal policies for both private and shared caches, regardless of cache size. This is mainly because even with large caches, there are still a significant number of both useful and useless prefetches generated. Therefore, the interference between prefetch and demand requests still needs to be intelligently controlled.

There are two other notable observations in Fig. 28: 1) the demand-prefetch-equal policy starts outperforming the demand-first policy for private caches greater than $1 \mathrm{MB}$ (8 MB for the shared cache), and 2) performance of APS (without APD) becomes closer to that of PADC (APS and APD together) as the cache size becomes larger. Note that these trends hold for both private and shared L2 caches.

Both observations can be explained by two reasons. First, a larger cache reduces irregular (or hard-to-prefetch) conflict cache misses due to the increased cache capacity. This makes the prefetcher more accurate because it reduces the allocations of stream entries for hard-to-prefetch access patterns (note that only a demand cache miss allocates a stream prefetch entry, as discussed in Section 2.3). Second, a larger cache can tolerate some degree of cache pollution. Due to the increased cache capacity, the probability of replacing a demand or useful prefetch line with a useless prefetch in the cache is reduced.

For these reasons, the effect of deprioritizing or dropping likely-useless prefetches becomes less significant with a larger cache. As a result, as cache size increases, techniques that prioritize demands (e.g., demand-first) and drop prefetches (APD) start becoming less effective. However, the interference between prefetch and demand requests is not completely eliminated since some applications still suffer from useless prefetches. PADC (and APS) is effective in reducing this interference in systems with large caches, and therefore, still performs significantly better than the rigid scheduling policies.

Note that PADC is cost-effective for both private and shared L2 caches. For instance, PADC with a $512 \mathrm{~KB}$ private L2 cache per core performs almost the same as demand-first with a $2 \mathrm{MB}$ private $\mathrm{L} 2$ cache per core, as shown in Fig. 28a. Thus, PADC (which requires only $4.25 \mathrm{~KB}$ storage) achieves the equivalent performance improvement that an additional $6 \mathrm{MB}(1.5 \mathrm{MB} \times 4$ cores) of cache storage would provide in the four-core system.

\subsection{Effect on Other Prefetching Mechanisms}

To show that the benefits of PADC are orthogonal to the prefetching algorithm employed, we briefly evaluate the effect of our PADC on different types of prefetchers: PCbased stride [1], C/DC [27], and the Markov prefetcher [9]. Fig. 29 shows performance and bus traffic results averaged over all 32 workloads run on the four-core system with the three different prefetchers. PADC consistently improves performance and reduces bandwidth consumption compared to the demand-first or demand-prefetch-equal policies with all three prefetchers.

The PC-based stride and C/DC prefetchers successfully capture a significant number of memory access patterns as the stream prefetcher does thereby increasing the potential for exploiting row buffer locality. In addition, these prefetchers also generate many useless prefetches for certain applications. Therefore, PADC significantly improves performance and bandwidth efficiency with these prefetchers by increasing DRAM throughput for useful requests and reducing the negative impact of useless prefetches.

The performance improvement of PADC on the Markov prefetcher is the least. This is because the Markov prefetcher, which exploits temporal as opposed to spatial correlation, does not work as well as the other prefetchers for the SPEC benchmarks. It generates many useless prefetches which lead to significant waste/interference in DRAM bandwidth, cache space, and memory queue resources. Furthermore, it does not generate many useful prefetches for the SPEC benchmarks, and therefore, its maximum potential for performance improvement is low. As such, the Markov prefetcher significantly increases bandwidth consumption and results in little performance improvement compared to no prefetching, as shown in Fig. 29b. PADC improves performance of the Markov prefetcher (mainly due to APD) by removing a large number of useless prefetches while keeping the small number of useful prefetches. PADC improves WS by 2.2 percent and reduces bandwidth consumption by 10.3 percent (mainly due to APD) compared to the demand-first policy. We conclude that PADC is effective with a wide variety of prefetching mechanisms. 


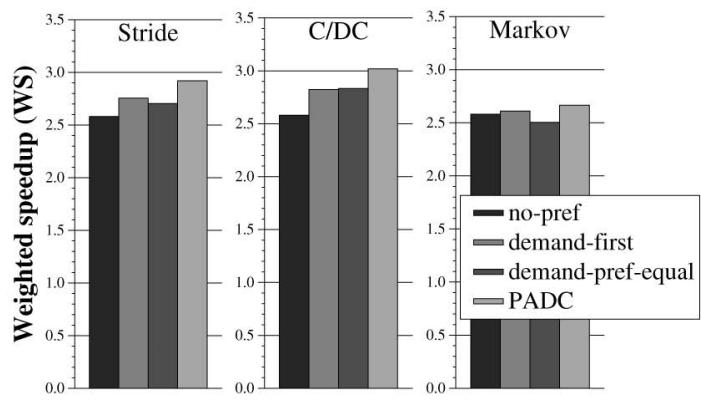

(a)

Fig. 29. PADC on stride, C/DC, and Markov prefetchers. (a) Performance.

\subsection{Comparison with Dynamic Data Prefetch Filtering and Feedback Directed Prefetching}

Dynamic Data Prefetch Filtering (DDPF) [46] tries to eliminate useless prefetches based on whether or not the prefetch was useful in the past. It records either the past usefulness of the prefetched address (or the PC of the instruction which triggered the prefetch) in a table similar to how a two-level branch predictor stores history information. When a prefetch request is created, the history table is consulted and the previous usefulness information is used to determine whether or not to send out the prefetch request. Feedback Directed Prefetching (FDP) [36] adaptively adjusts the aggressiveness of the prefetcher in order to reduce its negative effects.

Recall that PADC has two components: APS and APD. Both DDPF and FDP are orthogonal to APS because they do not deal with the scheduling of prefetches with respect to demands. As such, they can be employed together with APS to maximize the benefits of prefetching. On the other hand, the benefits of DDPF, FDP, and APD overlap. DDPF filters out useless prefetches before they are sent to the memory system. FDP eliminates useless prefetches by reducing the aggressiveness of the prefetcher thereby reducing the likelihood that useless prefetch requests are generated. In contrast, APD eliminates useless prefetches by dropping them after they are generated. As a result, we find (based on our experimental analyses) that APD has the following advantages over DDPF and FDP:

1. Both DDPF and FDP eliminate not only useless prefetches but also a significant fraction of useful prefetches. DDPF removes many useful prefetches by falsely predicting many useful prefetches to be useless. This is due to the aliasing problem caused by sharing the limited size of the history table among many addresses. FDP can eliminate useful prefetches when it reduces the aggressiveness of the prefetcher. In addition, we found that FDP can be very slow in increasing the aggressiveness of the prefetcher when a new phase starts execution. In such cases, FDP cannot issue useful prefetches, whereas APD would have issued them because it always keeps the prefetcher aggressive.

2. The hardware cost of DDPF for an L2 cache is expensive since each L2 cache line and MSHR must carry several bits for indexing the prefetch history table (PHT) to update the table appropriately. For

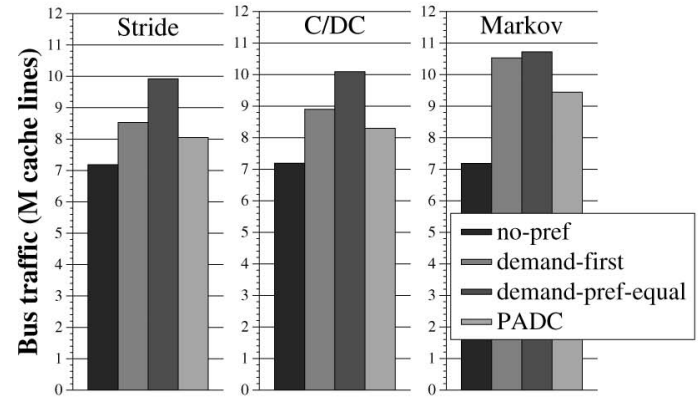

(b)

(b) Traffic

example, for a PC-based gshare DDPF with a 4 K-entry PHT, 24 bits (12-bit branch history and 12-bit load PC bits) per cache line are needed in addition to the prefetch bit per cache line. For the four-core system we use, this index information alone accounts for $96 \mathrm{~KB}$ of storage. In contrast, APD does not require significant hardware cost, as shown in Section 4.5.

3. FDP requires the tuning of multiple threshold values [36] to throttle the aggressiveness of the prefetcher which is a nontrivial optimization problem. APD allows the baseline prefetcher to always be very aggressive because it can eliminate useless prefetches after they are generated. As such, there is no need to tune multiple different threshold values in APD because the aggressiveness of the prefetcher never changes.

To evaluate performance of these mechanisms, we implemented DDPF (PC-based gshare DDPF for L2 cache prefetch filtering [46]) and FDP in our CMP system. All the relevant parameters (FDP: prefetch accuracy of 90 and 40 percent, lateness threshold of one percent, and pollution threshold of 0.5 percent, and pollution filter size of $4 \mathrm{Kbits}$; DDPF: filtering threshold of three and table size of $4 \mathrm{~K}$ entry 2-bit counters) for DDPF and FDP were tuned for the best performance with the stream prefetcher in our CMP system. Fig. 30 shows performance and bus traffic of different combinations of DDPF, FDP, and PADC averaged across the 32 workloads run on the four-core system. From left to right, the seven bars show:

1. baseline stream prefetching with the rigid demandfirst policy,

2. DDPF with demand-first policy,

3. FDP with demand-first policy,

4. APD with demand-first policy,

5. DDPF combined with APS,

6. FDP combined with APS, and

7. APD combined with APS (i.e., PADC).

When used with the demand-first policy, DDPF and FDP improve performance by 1.5 and 1.7 percent, respectively, while reducing bus traffic by 22.8 and 12.6 percent. In contrast, APD improves performance by 2.6 percent, while reducing bus traffic by 10.4 percent. DDPF and FDP eliminate more useless prefetches than APD resulting in less bus traffic. However, for the very same reason, DDPF and FDP eliminate many useful prefetches as well. Therefore, their performance improvement is not as high as APD. 


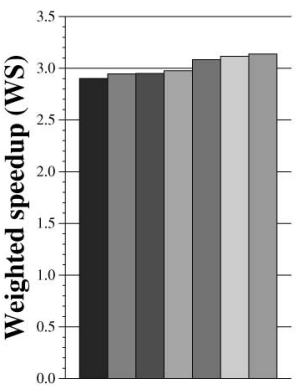

(a)

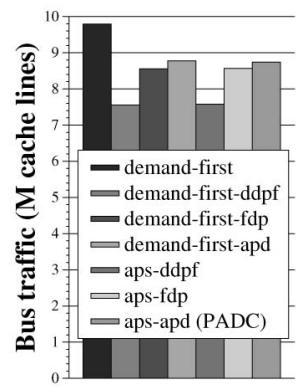

(b)

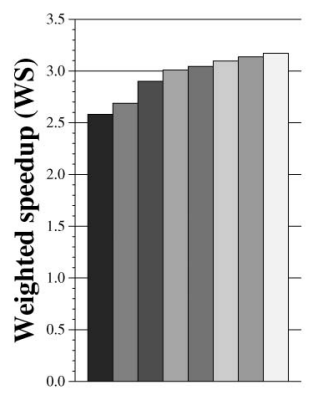

(a)

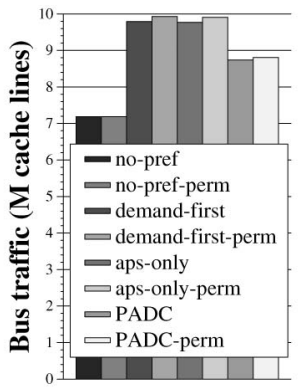

(b)
Fig. 30. Comparison with DDPF and FDP with demand-first scheduling. (a) Performance. (b) Average traffic.

Our adaptive scheduling policy and DDPF/FDP are complementary and improve performance significantly when combined together. When used together with Adaptive Prefetch Scheduling, DDPF and FDP improve performance by 6.3 and 7.4 percent, respectively. Finally, the results show that PADC outperforms the combination of DDPF/FDP and APS which illustrates that Adaptive Prefetch Dropping is better suited to eliminate the negative performance effects of prefetching than DDPF and FDP. We conclude that 1 ) our adaptive scheduling technique complements DDPF and FDP, whereas our APD technique outperforms DDPF and FDP, and 2) DDPF and FDP reduce bandwidth consumption more than APD, but they do so at the expense of performance.

If a prefetch filtering mechanism is able to eliminate all useless prefetches while keeping all useful prefetches, the demand-prefetch-equal policy would be best performing. That is to say we do not need an adaptive memory scheduling policy since all prefetches sent to the memory system would be useful. However, it is not trivial to design such a perfect prefetch filtering mechanism. As discussed above, DDPF and FDP filter out not only useless prefetches but also a lot of useful prefetches. Therefore, combining those schemes with demand-prefetch-equal does not necessarily significantly improve performance since the benefits of useful prefetches are reduced.

Fig. 31 shows performance and average traffic when DDPF and FDP are combined with demand-prefetch-equal. Since DDPF and FDP remove a significant number of useful prefetches, performance improvement is not very significant (only by 2.3 and 2.7 percent compared to demandfirst). On the other hand, PADC significantly improves

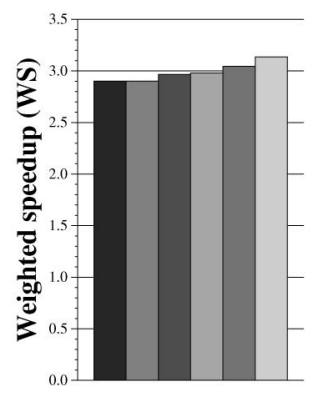

(a)

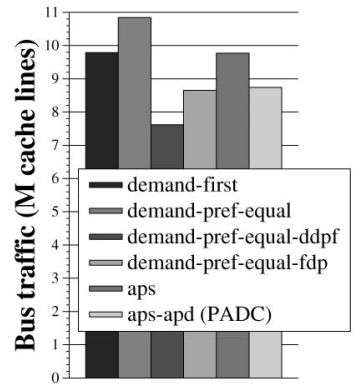

(b)
Fig. 31. Comparison to DDPF and FDP with demand-prefetch-equal scheduling. (a) Performance. (b) Average traffic.
Fig. 32. Effect on permutation-based page interleaving. (a) Performance. (b) Average traffic.

performance (by 8.2 percent) by keeping the benefits of useful prefetches as much as possible.

\subsection{Interaction with Permutation-Based Page Interleaving}

Permutation-based page interleaving [43] aims to reduce row-conflicts by randomly mapping the DRAM bank indexes of addresses so that they are more spread out across the multiple banks in the memory system. This technique significantly improves DRAM throughput by increasing utilization of multiple DRAM banks. The increased utilization of the banks has the potential to reduce the interference between memory requests. However, this technique cannot completely eliminate the interference between demand and prefetch requests in the presence of prefetching. Any rigid prefetch scheduling policy in conjunction with this technique will still have the same problem we describe in Section 3: none of the rigid prefetch scheduling policies can achieve the best performance for all applications since they are not aware of prefetch usefulness. Therefore, PADC is complementary to permutation-based page interleaving.

Fig. 32 shows performance impact of PADC for the 32 fourcore workloads when a permutation-based interleaving scheme is applied. The permutation-based scheme improves the system performance by 3.8 percent over our baseline with the demand-first policy. This is because the permutation scheme reduces row-conflicts by spreading out requests across multiple banks. APS and PADC consistently work effectively combined with the permutation-based interleaving scheme by efficiently managing the interference between demands and prefetches based on usefulness of prefetches. APS and PADC improve system performance by 2.9 and 5.4 percent, respectively, compared to the demand-first policy with the permutation-based interleaving scheme. Also, $\mathrm{PADC}$ reduces bandwidth consumption by 11.3 percent due to adaptive prefetch dropping.

\subsection{Effect on a Runahead Execution Processor}

Runahead execution [24] is a promising technique that prefetches useful data by executing future instructions that are independent of a long latency (runahead-causing) load instruction during the stall time of the load instruction. Because it is based on the execution of actual instructions, runahead execution can prefetch irregular data access patterns as well as regular ones. Usually, runahead execution complements hardware prefetching and results in high 


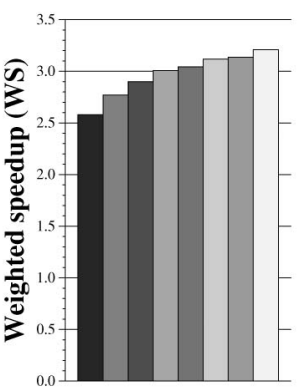

(a)

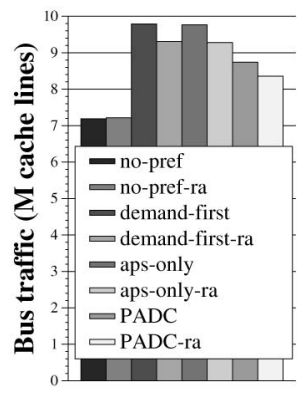

(b)
Fig. 33. Effect on runahead execution. (a) Performance. (b) Average traffic.

performance. In this section, we analyze the effect of PADC on a runahead processor. We implemented runahead capability in our baseline system by augmenting invalid bits in the register files for each core. Since memory requests during runahead modes are very accurate most of the time [24], we treat runahead requests the same as demand requests in DRAM scheduling.

Fig. 33 shows the effect of PADC on a runahead processor for the 32 workloads on the four-core CMP system. Each runahead processor has exactly the same parameters as our baseline processor, but it also uses a 512-byte runahead cache to support store load forwarding during runahead execution. Adding runahead execution on top of the baseline demand-first policy improves system performance by 3.7 percent and also reduces bandwidth consumption by 5.0 percent. This is because we use a prefetcher update policy that trains existing stream prefetch entries but does not allocate a new stream prefetch entry on a cache miss during runahead execution (only-train). Previous research [21] shows that this policy is best performing and most efficient. Runahead execution with the only-train policy can make prefetching more accurate and efficient by capturing irregular cache misses during runahead execution. These irregular misses train existing stream prefetch entries, but new and more speculative stream prefetch entries will not be created during runahead mode. This not only prevents the prefetcher from generating useless prefetches due to falsely created streams but also improves the accuracy and timeliness of the stream prefetcher since existing streams continue to be trained during runahead mode.

Fig. 33 shows that PADC still effectively improves performance by 6.7 percent and reduces bandwidth consumption by 10.2 percent compared to a runahead CMP processor with the stream prefetcher and the demand-first policy. We conclude that PADC is effective at improving performance and bandwidth efficiency for an aggressive runahead CMP by successfully reducing the interference between demand/runahead and prefetch requests in the DRAM controller.

\section{Related Work}

The main contribution of our work beyond previous research is an adaptive way of handling prefetch requests in the memory controller's scheduling and buffer management policies. To our knowledge, none of the previously proposed DRAM controllers adaptively prioritize between prefetch and demand requests nor do they adaptively drop useless prefetch requests based on prefetch usefulness information obtained from the prefetcher. We discuss closely related work in DRAM scheduling, prefetch filtering, and adaptive prefetching.

\subsection{Prefetch Handling in DRAM Controllers}

Many previous DRAM scheduling policies were proposed to improve DRAM throughput in single-threaded [47], [31], [7], multithreaded [30], [25], [44], and stream-based [17], [42] systems. In addition, several recent works [26], [22], [23] proposed techniques for fair DRAM scheduling across different applications sharing the DRAM system. Some of these previous proposals [47], [31], [25], [44], [26], [22], [23] do not discuss how prefetch requests are handled with respect to demand requests. Therefore, our mechanism is orthogonal to these scheduling policies. These policies can be extended to adaptively prioritize between demand and prefetch requests and to adaptively drop useless prefetch requests.

The remaining DRAM controller proposals take two different approaches to handling prefetch requests. First, some proposals [14], [7], [8] always prioritize demand requests over prefetch requests. Other proposals [42], [30], including some memory controllers in existing processors [10], [38], treat prefetch requests the same as demand requests. As such, these previous DRAM controller proposals handle prefetch requests rigidly. As we have shown in Sections 1 and 3, rigid handling of prefetches can cause significant performance loss compared to adaptive prefetch handling. Our work improves upon these proposals by incorporating the effectiveness of prefetching into DRAM scheduling decisions.

\subsection{Prefetch Filtering}

Our APD scheme shares the same goal of eliminating useless prefetches with several other previous proposals. However, our mechanism provides either higher bandwidth efficiency or better adaptivity compared to these works.

Charney and Puzak [2] and Mutlu et al. [20] proposed prefetch filtering mechanisms using on-chip caches (using the tag store). Both of these proposals unnecessarily consume memory bandwidth since useless prefetches are filtered out only after they are serviced by the DRAM system. In contrast, APD eliminates useless prefetches before they consume valuable DRAM bandwidth.

Mowry et al. [19] proposed a prefetch dropping mechanism that cancels software prefetches when the prefetch issue queue is full to avoid processor stall. On the other hand, our scheme drops hardware prefetch requests only if their age is greater than a dynamically adjusted threshold (based on prefetch accuracy). Srinivasan et al. [37] use a profiling technique to mark load instructions that are likely to generate useful prefetches. This mechanism needs ISA support to mark the selected load instructions and cannot adapt to phase behavior in prefetcher accuracy. In contrast, APD does not require ISA changes and can adapt to changes in prefetcher accuracy.

Zhuang and Lee [45], [46] propose a mechanism that eliminates the prefetch request for an address if the prefetch request for the same address was useless in the past. PADC outperforms and also complements their mechanism as discussed in Section 6.12. 


\subsection{Adaptive Prefetching}

Several previous works proposed changing the aggressiveness of the hardware prefetcher based on dynamic information. Our work is either complementary to or higher performing than these proposals, as described below.

Hur and Lin [8] designed a probabilistic prefetching technique which adjusts prefetcher aggressiveness. They also schedule prefetch requests to DRAM adaptively based on the frequency of demand request DRAM bank conflicts caused by prefetch requests. However, their scheme always prioritizes demand requests over prefetches. In contrast, our mechanism adapts the prioritization policy between demands and prefetches based on prefetcher accuracy. As a result, Hur and Lin's proposal can be combined with our adaptive prefetch scheduling policy to provide even higher performance.

Srinath et al. [36] show how adjusting the aggressiveness of the prefetcher based on accuracy, lateness, and cache pollution information can reduce bus traffic without compromising the benefit of prefetching. As shown in Section 6.12, PADC outperforms and also complements their mechanism.

\section{Conclusion}

This paper shows that existing DRAM controllers that employ rigid, nonadaptive prefetch scheduling and buffer management policies cannot achieve the best performance since they do not take into account the usefulness of prefetch requests. To overcome this limitation, we propose a low hardware cost Prefetch-Aware DRAM Controller, which aims to 1) maximize the benefit of useful prefetches by adaptively prioritizing them and 2) minimize the harm caused by useless prefetches by adaptively deprioritizing and dropping them from the memory request buffers. To this end, PADC dynamically adapts its memory scheduling and buffer management policies based on prefetcher accuracy. Our evaluation shows that PADC significantly improves system performance and bandwidth efficiency on both single-core and multicore systems. We conclude that incorporating awareness of prefetch usefulness into memory controllers is critical to efficiently utilizing valuable memory system resources in current and future systems.

\section{ACKNOWLEDGMENTS}

Many thanks to Minsik Cho, Viji Srinivasan, José A. Joao, Eiman Ebrahimi and other HPS members. We also thank the anonymous reviewers for their comments. Chang Joo Lee and Veynu Narasiman were supported by IBM and NVIDIA PhD fellowships respectively during this work. We gratefully acknowledge the support of the Cockrell Foundation, Intel, AMD, and Gigascale Systems Research Center. This research was partially supported by NSF CAREER Award CCF-0953246.

\section{REFERENCES}

[1] J. Baer and T. Chen, "An Effective On-Chip Preloading Scheme to Reduce Data Access Penalty," Proc. ACM/IEEE Conf. Supercomputing (Supercomputing '91), pp. 178-186, 1991.

[2] M. Charney and T. Puzak, "Prefetching and Memory System Behavior of the SPEC95 Benchmark Suite," IBM J. Research and Development, vol. 31, no. 3, pp. 265-286, 1997.
[3] V. Cuppu, B. Jacob, B. Davis, and T. Mudge, "A Performance Comparison of Contemporary DRAM Architectures," Proc. 26th Ann. Int'l Symp. Computer Architecture (ISCA-26), pp. 222-233, 1999.

[4] S. Eyerman and L. Eeckhout, "System-Level Performance Metrics for Multiprogram Workloads," IEEE Micro, vol. 28, no. 3, pp. 42-53, May 2008.

[5] J.D. Gindele, "Buffer Block Prefetching Method," IBM Technical Disclosure Bull., vol. 20, no. 2, pp. 696-697, July 1977.

[6] G. Hinton, D. Sager, M. Upton, D. Boggs, D. Carmean, A. Kyker, and P. Roussel, "The Microarchitecture of the Pentium 4 Processor," Intel Technology J., vol. 1, p. 2001, Feb. 2001.

[7] I. Hur and C. Lin, "Adaptive History-Based Memory Scheduler," Proc. 37th Ann. IEEE/ACM Int'l Symp. Microarchitecture (MICRO-37), 2004.

[8] I. Hur and C. Lin, "Memory Prefetching Using Adaptive Stream Detection," Proc. 39th Ann. IEEE/ACM Int'l Symp. Microarchitecture (MICRO-39), 2006.

[9] D. Joseph and D. Grunwald, "Prefetching Using Markov Predictors," Proc. 24th Ann. Int'l Symp. Computer Architecture (ISCA-24), pp. 252-263, 1997.

[10] R. Kalla, B. Sinharoy, and J.M. Tendler, "IBM Power5 Chip: A Dual-Core Multithreaded Processor," IEEE Micro, vol. 24, no. 2, pp. 40-47, Mar./Apr. 2004.

[11] T. Karkhanis and J.E. Smith, "A Day in the Life of a Data Cache Miss," Proc. Second Workshop Memory Performance Issues, 2002.

[12] H.Q. Le, W.J. Starke, J.S. Fields, F.P. O'Connell, D.Q. Nguyen, B.J. Ronchetti, W.M. Sauer, E.M. Schwarz, and M.T. Vaden, "IBM Power6 Microarchitecture," IBM J. Research and Development, vol. 51, pp. 639-662, 2007.

[13] C.J. Lee, O. Mutlu, V. Narasiman, and Y.N. Patt, "Prefetch-Aware DRAM Controllers," Proc. 41st Ann. IEEE/ACM Int'l Symp. Microarchitecture (MICRO-41), 2008.

[14] W.-F. Lin, S.K. Reinhardt, and D. Burger, "Reducing DRAM Latencies with an Integrated Memory Hierarchy Design," Proc. Seventh Int'l Symp. High-Performance Computer Architecture (HPCA-7), pp. 301-312, 2001.

[15] K. Luo, J. Gummaraju, and M. Franklin, "Balancing Throughput and Fairness in SMT Processors," Proc. IEEE Int'l Symp. Performance Analysis of Systems and Software (ISPASS), pp. 164171, 2001.

[16] S. McFarling, "Combining Branch Predictors," Technical Report TN-36, Digital Western Research Laboratory, June 1993.

[17] S.A. McKee, W.A. Wulf, J.H. Aylor, R.H. Klenke, M.H. Salinas, S.I. Hong, and D.A. Weikle, "Dynamic Access Ordering for Streamed Computations," IEEE Trans. Computers, vol. 49, no. 11, pp. 1255-1271, Nov. 2000.

[18] Micron, 2Gb DDR3 SDRAM, MT41J512M4-64 Meg $x 4 x 8$ Banks, http://download.micron.com/pdf/datasheets/dram/ddr3/, 2009.

[19] T.C. Mowry, M.S. Lam, and A. Gupta, "Design and Evaluation of a Compiler Algorithm for Prefetching," Proc. Fifth Int'l Conf. Architectural Support for Programming Languages and Operating Systems, pp. 62-73, 1992.

[20] O. Mutlu, H. Kim, D.N. Armstrong, and Y.N. Patt, "Using the First-Level Caches as Filters to Reduce the Pollution Caused by Speculative Memory References," Int'l J. Parallel Programming, vol. 33, no. 5, pp. 529-559, Oct. 2005.

[21] O. Mutlu, H. Kim, and Y.N. Patt, "Techniques for Efficient Processing in Runahead Execution Engines," Proc. Ann. 32nd Int'l Symp. Computer Architecture (ISCA-32), 2005.

[22] O. Mutlu and T. Moscibroda, "Stall-Time Fair Memory Access Scheduling for Chip Multiprocessors," Proc. 40th Ann. IEEE/ACM Int'l Symp. Microarchitecture (MICRO-40), 2007.

[23] O. Mutlu and T. Moscibroda, "Parallelism-Aware Batch Scheduling: Enhancing Both Performance and Fairness of Shared DRAM Systems," Proc. 35th Ann. Int'l Symp. Computer Architecture (ISCA-35), 2008.

[24] O. Mutlu, J. Stark, C. Wilkerson, and Y.N. Patt, "Runahead Execution: An Alternative to Very Large Instruction Windows for Out-of-Order Processors," Proc. Ninth Int'l Symp. High-Performance Computer Architecture (HPCA-9), 2003.

[25] C. Natarajan, B. Christenson, and F. Briggs, "A Study of Performance Impact of Memory Controller Features in MultiProcessor Server Environment," Proc. Workshop Memory performance Issues (WMPI), pp. 80-87, 2004. 
[26] K.J. Nesbit, N. Aggarwal, J. Laudon, and J.E. Smith, "Fair Queuing Memory Systems," Proc. 39th Ann. IEEE/ACM Int'l Symp. Microarchitecture (MICRO-39), 2006.

[27] K.J. Nesbit, A.S. Dhodapkar, J. Laudon, and J.E. Smith, "AC/DC: An Adaptive Data Cache Prefetcher," Proc. 13th Int'l Conf. Parallel Architecture and Compilation Techniques (PACT-13), 2004.

[28] H. Patil, R. Cohn, M. Charney, R. Kapoor, A. Sun, and A Karunanidhi, "Pinpointing Representative Portions of Large Intel Itanium Programs with Dynamic Instrumentation," Proc. 37th Ann. IEEE/ACM Int'l Symp. Microarchitecture (MICRO-37), 2004.

[29] M.K. Qureshi, D.N. Lynch, O. Mutlu, and Y.N. Patt, "A Case for MLP-Aware Cache Replacement," Proc. 33rd Ann. Int'l Symp. Computer Architecture (ISCA-33), 2006.

[30] S. Rixner, "Memory Controller Optimizations for Web Servers," Proc. 37th Ann. IEEE/ACM Int'l Symp. Microarchitecture (MICRO-37), 2004

[31] S. Rixner, W.J. Dally, U.J. Kapasi, P. Mattson, and J.D. Owens, "Memory Access Scheduling," Proc. 27th Ann. Int'l Symp. Computer Architecture (ISCA-27), 2000.

[32] A.J. Smith, "Cache Memories," Computing Surveys, vol. 14, no. 4, pp. 473-530, 1982.

[33] W.E. Smith, "Various Optimizers for Single Stage Production," Naval Research Logistics Quarterly, vol. 3, pp. 59-66, 1956.

[34] A. Snavely and D.M. Tullsen, "Symbiotic Job Scheduling for a Simultaneous Multithreading Processor," Proc. Ninth Int'l Conf. Architectural Support for Programming Languages and Operating Systems (ASPLOS-9), pp. 164-171, 2000.

[35] L. Spracklen and S.G. Abraham, "Chip Multithreading: Opportunities and Challenges," Proc. 11th Int'l Symp. High-Performance Computer Architecture (HPCA-11), pp. 248-252, 2005.

[36] S. Srinath, O. Mutlu, H. Kim, and Y.N. Patt, "Feedback Directed Prefetching: Improving the Performance and Bandwidth-Efficiency of Hardware Prefetchers," Proc. 13th Int'l Symp. HighPerformance Computer Architecture (HPCA-13), 2007.

[37] V. Srinivasan, G.S. Tyson, and E.S. Davidson, "A Static Filter for Reducing Prefetch Traffic," Technical Report CSE-TR-400-99, Univ. of Michigan, 1999.

[38] Sun Microsystems, Inc., OpenSPARC T1 Microarchitecture Specification

[39] J. Tendler, S. Dodson, S. Fields, H. Le, and B. Sinharoy, "POWER4 System Microarchitecture," IBM Technical White Paper, Oct. 2001.

[40] O. Wechsler, "Inside Intel Core Microarchitecture," Intel Technical White Paper, 2006

[41] T.-Y. Yeh and Y.N. Patt, "Alternative Implementations of TwoLevel Adaptive Branch Prediction," Proc. 19th Ann. Int'l Symp. Computer Architecture (ISCA-19), 1992.

[42] C. Zhang and S.A. McKee, "Hardware-Only Stream Prefetching and Dynamic Access Ordering," Proc. 14th Int'l Conf. Supercomputing (ICS-14), 2000.

[43] Z. Zhang, Z. Zhu, and X. Zhang, "A Permutation-Based Page Interleaving Scheme to Reduce Row-Buffer Conflicts and Exploit Data Locality," Proc. 27th Ann. Int'l Symp. Computer Architecture (ISCA-27), 2000.

[44] Z. Zhu and Z. Zhang, "A Performance Comparison of DRAM Memory System Optimizations for SMT Processors," Proc. 11th Int'l Symp. High-Performance Computer Architecture (HPCA-11), 2005

[45] X. Zhuang and H.-H.S. Lee, "A Hardware-Based Cache Pollution Filtering Mechanism for Aggressive Prefetches," Proc. 32nd Int'l Conf. Parallel Processing, pp. 286-293, 2003.

[46] X. Zhuang and H.-H.S. Lee, "Reducing Cache Pollution via Dynamic Data Prefetch Filtering," IEEE Trans. Computers, vol. 56, no. 1, pp. 18-31, Jan. 2007.

[47] W. Zuravleff and T. Robinbson, "Controller for a Synchronous DRAM That Maximizes Throughput by Allowing Memory Requests and Commands to be Issued Out of Order," US Patent Number 5,630,096, 1997.

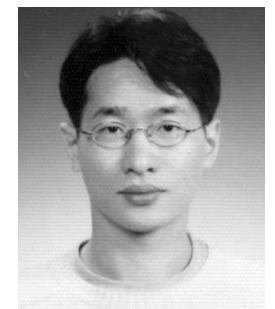

Chang Joo Lee received the BS degree in electrical engineering from Seoul National University in 2001, and the MS degree in computer engineering in 2004 from the University of Texas at Austin, where he is currently working toward the $\mathrm{PhD}$ degree in computer engineering. $\mathrm{He}$ is interested in computer architecture research mainly focused on high-performance memory systems and energy-efficient microarchitectures. He was a recipient of the scholarship from the Ministry of Information and Communication in Korea during 2002-2006, the IBM PhD fellowship in 2007, and the IBM scholarship in 2008. He is a student member of the IEEE.

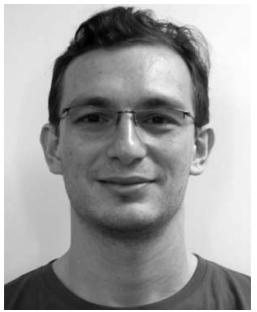

Onur Mutlu received the BS degrees in computer engineering and psychology from the University of Michigan, Ann Arbor, and the MS and $\mathrm{PhD}$ degrees in electrical and computer engineering from the University of Texas at Austin. $\mathrm{He}$ is an assistant professor of electrical and computer engineering at Carnegie Mellon University. He is interested in computer architecture and systems research. Prior to Carnegie Mellon, he worked at Microsoft Research, Intel Corporation, and Advanced Micro Devices. He received the University of Texas George H. Mitchell Award for Excellence in Graduate Research in 2005, Microsoft Gold Star Award in 2008, US National Science Foundation (NSF) CAREER Award in 2010, and several computer architecture top pick paper awards from the IEEE Micro. He is a member of the IEEE.

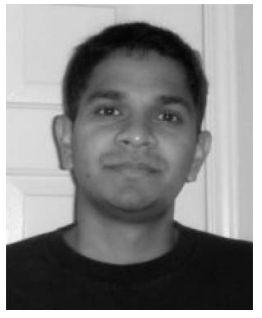

Veynu Narasiman received the BS degree in electrical/computer engineering, the BA degree in plan II in 2004, and the MS degree in computer engineering in 2006 from the University of Texas at Austin, where he is currently working toward the $\mathrm{PhD}$ degree. His research interests in computer architecture focus on general-purpose graphics processing units (GPGPUs) and high-performance memory systems. As a student, he has received a number of awards including a National Merit Scholarship, the Microelectronics and Computer Development Fellowship, and the NVIDIA PhD Fellowship. $\mathrm{He}$ is a student member of the IEEE.

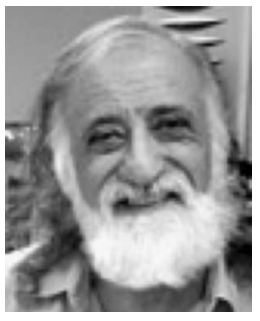

Yale N. Patt received the BS degree in electrical engineering from Northeastern, and the MS and $\mathrm{PhD}$ degrees in electrical engineering from Stanford. He is the Ernest Cockrell, Jr., centennial chair in engineering and a professor of electrical and computer engineering at Texas. He continues to thrive on teaching the large (400 students) freshman introductory course in computing and advanced graduate courses in microarchitecture, directing the research of eight $\mathrm{PhD}$ students, and consulting in the microprocessor industry. He is the coauthor of Introduction to Computing Systems: from bits and gates to C and beyond, McGraw-Hill (second edition, 2004), his preferred approach to introducing freshmen to computing. He has received a number of awards for his research and teaching, including the IEEE/ACM EckertMauchly Award for his research in microarchitecture and the ACM Karl V. Karlstrom Award for his contributions to education. He is a fellow of the IEEE. More detail about his research can be found at http:// www.ece.utexas.edu/ patt.

$\triangleright$ For more information on this or any other computing topic, please visit our Digital Library at www.computer.org/publications/dlib. 